\title{
¿Variability of Soil Moisture and Sea Surface Temperatures Similarly Important for Warm-Season Land Climate in the Community Earth System Model $\mathscr{O}$
}

\author{
Rene Orth AND Sonia I. SENEVIRATne \\ Institute for Atmospheric and Climate Science, ETH Zurich, Zurich, Switzerland
}

(Manuscript received 12 August 2015, in final form 24 November 2016)

\begin{abstract}
Both sea surface temperatures (SSTs) and soil moisture (SM) can influence climate over land. This paper presents a comprehensive comparison of SM versus SST impacts on land climate in the warm season. The authors perform fully coupled ensemble experiments with the Community Earth System Model in which they prescribe SM or SSTs to the long-term median seasonal cycles. It is found that SM variability overall impacts warm-season land climate to a similar extent as SST variability, in the midlatitudes, tropics, and subtropics. Removing SM or SST variability impacts land climate means and reduces land climate variability at different time scales by $10 \%-50 \%$ (temperature) and $0 \%-10 \%$ (precipitation). Both SM- and SST-induced changes are strongest for hot temperatures (up to 50\%) and for extreme precipitation (up to 20\%). These results are qualitatively similar for the present day and the end of the twenty-first century. Removed SM variability affects surface climate through corresponding variations in surface energy fluxes, and this is controlled to first order by the land-atmosphere coupling strength and the natural SM variability. SST-related changes are partly controlled by the relation of local temperature or precipitation with the El Niño-Southern Oscillation. In addition, in specific regions SST-induced SM changes alter the "direct" SST-induced climate changes; on the other hand, SM variability is found to slightly affect SSTs in some regions. Nevertheless a large level of independence is found between SM-climate and SST-climate coupling. This highlights the fact that SM conditions can influence land climate variables independently of any SST effects and that (initial) soil moisture anomalies can provide valuable information in (sub)seasonal weather forecasts.
\end{abstract}

\section{Introduction}

It is well known that the surface temperature of the world's oceans influences climate. There is a local impact on ocean climate, but there are also effects on large-scale atmospheric circulation and wave patterns and, hence, eventually on land climate. Such teleconnections are especially active in tropical and subtropical regions. The most well-known example is the El Niño-Southern Oscillation (ENSO; Rasmusson and Carpenter 1982), which

Denotes content that is immediately available upon publication as open access.

Supplemental information related to this paper is available at the Journals Online website: http://dx.doi.org/10.1175/ JCLI-D-15-0567.s1.

Corresponding author e-mail: Rene Orth, rene.orth@env.ethz.ch relates sea surface temperatures (SSTs) in the eastern tropical Pacific Ocean to temperature and precipitation anomalies in many regions across different continents.

Another important control of (mostly warm season) climate over land is the available moisture in the soil [see, e.g., Seneviratne et al. (2010) for a review]. Soil moisture (SM) may alter the partitioning of incoming net radiation into the sensible and latent heat fluxes. For instance, soil that is drier than average limits evaporation over bare soil and transpiration of plants, which adapt their behavior to moisture availability (Teuling et al. 2009). Therefore SM has implications for several aspects of the surface climate such as temperature (Hirschi et al. 2011; Mueller and Seneviratne 2012; Whan et al. 2015), moisture availability in the lower atmosphere, boundary layer stability (Ek and Holtslag 2004), and eventually also precipitation (Koster et al. 2004; Taylor et al. 2011; Guillod et al. 2015). Whereas SST is a largely external control on land climate, SM is influenced by the atmospheric forcing and influences 
climate through its feedbacks on evapotranspiration and temperature (Seneviratne et al. 2010; Zscheischler et al. 2015). In this study, we assess and compare the influence of these two controls on land climate: that is, we consider (i) remotely induced effects of SSTs on large-scale circulation and heat/moisture advection on land and (ii) atmospheric impacts of variations in land-atmosphere exchanges of moisture and heat, which can be due to local but also to nonlocal effects of soil moisture (e.g., due to modified circulation patterns or changes in advected heat or moisture).

Besides their impact on climate, there is a remarkable predictability associated with both SM (Koster and Suarez 2001; Orth and Seneviratne 2013) and SST (Jin and Kinter 2009), which may extend to weeks or months. Hence, they may contribute substantial skill in weather and climate forecasting (Shukla 1998; Koster et al. 2011; Orth and Seneviratne 2014). However, memory of SM or SST is only translated into weather and climate predictability if SM and/or SST have an influence on land climate means and variability (e.g., Seneviratne et al. 2006b). This interplay between predictability and influence on climate may vary considerably across regions, seasons, and also (forecasting) time scales; the challenge to understand these spatiotemporal effects is a main motivation for past studies and also for our analysis.

An early effort to compare land and ocean impacts on climate was made in a landmark study by Koster and Suarez (1995). They performed experiments with a global climate model (GCM) with prescribed SSTs and evaporation efficiencies (the ratios of evaporation to potential evaporation), respectively, and found that annual precipitation variability was overall more impacted by the latter. In a follow-up study with an updated model version that represented land-ocean teleconnections, and using an ensemble approach, Koster et al. (2000) found similar effects of land and ocean on precipitation variability. Furthermore they introduced the hypothesis that land-atmosphere interactions would, to first order, linearly amplify precipitation variability induced by the atmosphere and the ocean, which implies additivity of the precipitation variance induced by land, ocean, and atmosphere. This additivity hypothesis, however, could not be rigorously proved, and in a similar analysis by Reale et al. (2002) it was furthermore found not applicable in general. Moreover, that latter study compared land and ocean impacts at different time scales on overall precipitation variability, finding that annual variations in evaporation and SSTs are most important. Reale and Dirmeyer (2002) also reported comparable impacts of land and ocean on precipitation, confirming the results of Koster et al. (2000), even though they also showed important regional differences. Moving beyond precipitation by analyzing the impacts of land and ocean on climate variability in general, Dirmeyer (2001) shows an overall more important role of the SSTs, while recognizing the strong model dependency, especially in the representation of landatmosphere interactions. Based on these results, Dirmeyer (2003) and Douville (2003) pointed out an important role of (initial) soil moisture in (sub)seasonal forecasting.

In this study, we revisit the comparison of the effects of SSTs and SM on climate over land during the warm season. For this purpose, we perform fully coupled oceanland-atmosphere simulations with a state-of-the-art Earth system model (ESM) in which we remove the variability of SST and SM, respectively, by prescribing the long-term median seasonal cycles. We assess the related effects in a comprehensive framework in which we consider impacts on means and variabilities of temperature and precipitation. Besides using a state-of-the-art ESM with fully coupled ocean, land, and atmosphere, we complement the analyses from the previous studies by (i) studying impacts of SSTs and SM on different deciles of temperature and precipitation (i.e., also considering extremes in addition to mean changes), (ii) assessing the responses in both present and future climate comparing the results in present versus future climate, and (iii) comparing our results with output from a multimodel experiment with similar setup for prescribing SM (Seneviratne et al. 2013). While the amplification of atmosphere- or oceaninduced precipitation variability by the land was found not applicable in other studies, we revisit the additivity hypothesis proposed in Koster et al. (2000) and show that, in contrast to the amplification behavior, the implied additivity of SM and SST effects can be identified. This is done by comparing changes in climate in response to removed variability in SSTs or SM with corresponding changes in response to removed variability in SSTs and SM. Analyzing the combined effects of SM and SSTs is furthermore relevant, as they may lead to extreme events such as droughts (Hong and Kalnay 2000; Schubert et al. 2004; Wu and Kinter 2009).

\section{Methodology}

\section{a. Model description}

We employ in this study the Community Earth System Model (CESM; Gent et al. 2011), version 1.04. It is a state-of-the-art GCM that includes fully coupled atmosphere (Community Atmosphere Model, version 4; Neale et al. 2010), land (Community Land Model, version 4; Oleson et al. 2010), ocean (Parallel Ocean Program, version 2; Smith et al. 2010), and ice modules. In the land module, we activate the carbon-nitrogen cycle 
TABLE 1. Summary of performed model experiments. The three focal experiments for this study; REF, noSMvar, and noOCNvar, are highlighted in bold.

\begin{tabular}{|c|c|c|c|}
\hline Expt & Ocean $(\mathrm{SST}+$ sea ice $)$ & Soil (moisture + ice $)$ & Ensemble size \\
\hline REF & Interactive & Interactive & $\begin{array}{l}\text { Present: } 10 \text { simulations } \times 10 \mathrm{yr} \\
\text { Future: } 10 \text { simulations } \times 10 \mathrm{yr}\end{array}$ \\
\hline noSMvar & Interactive & Prescribed (median from REF) & $\begin{array}{l}\text { Present: } 10 \text { simulations } \times 10 \mathrm{yr} \\
\text { Future: } 10 \text { simulations } \times 10 \mathrm{yr}\end{array}$ \\
\hline noOCNvar & Prescribed (median from REF) & Interactive & $\begin{array}{l}\text { Present: } 10 \text { simulations } \times 10 \mathrm{yr} \\
\text { Future: } 10 \text { simulations } \times 10 \mathrm{yr}\end{array}$ \\
\hline noOCNvar_neutral & Prescribed (most neutral year from REF) & Interactive & Present: 10 simulations $\times 10 \mathrm{yr}$ \\
\hline noALLvar & Prescribed (median from REF) & Prescribed (median from REF) & $\begin{array}{l}\text { Present: } 10 \text { simulations } \times 10 \mathrm{yr} \\
\text { Future: } 10 \text { simulations } \times 10 \mathrm{yr}\end{array}$ \\
\hline noSMvar* & Prescribed (as in REF) & Prescribed (median from REF) & $\begin{array}{l}\text { Present: } 10 \text { simulations } \times 10 \mathrm{yr} \\
\text { Future: } 10 \text { simulations } \times 10 \mathrm{yr}\end{array}$ \\
\hline noOCNvar* & Prescribed (median from REF) & Prescribed (as in REF) & $\begin{array}{l}\text { Present: } 10 \text { simulations } \times 10 \mathrm{yr} \\
\text { Future: } 10 \text { simulations } \times 10 \mathrm{yr}\end{array}$ \\
\hline
\end{tabular}

but not the spatially dynamic vegetation, where species can propagate into new regions and/or retreat from others. In the atmospheric model we use a finite-volume dynamical core and shallow (Hack 1994) and deep convection schemes (Neale et al. 2008). CESM's landatmosphere coupling compares well with other climate models in terms of temporal and spatial structure even though it is relatively weak (Dirmeyer et al. 2013b; Seneviratne et al. 2013). We use a spatial resolution of approximately $2^{\circ}$ over land $\left(1.9^{\circ} \times 2.5^{\circ}\right)$ and approximately $1^{\circ}$ over the oceans (gx1v6) to perform 10 fully interactive ensemble simulations ranging from 1850 to 2100. These simulations are restarted (branched) from a preindustrial control simulation performed with the same setup, each at least 20 years apart from the next simulation to ensure different initial conditions and consequently independent realizations of the climate. In addition, we restart simulations in 1996 and 2091 from each ensemble member with identical setups, except that we prescribe SM and SST, respectively, and integrate each of them over 10 years (see section $2 \mathrm{~b}$ ). The model is driven with historical forcing (e.g., aerosols, greenhouse gases, and ozone) until 2005 and with forcing from the representative concentration pathway 8.5 (RCP8.5) scenario afterward. This ensemble of simulations serves as a reference to which we compare further ensemble simulations with prescribed SM and/or SSTs. For all simulations with interactive ocean, we employ the B_1850-2000_CN (1850 2005) [all active components, 1850 to 2000 transient, with CN (Carbon Nitrogen)] and B_RCP8.5_CN (2006-2100) (all active components, RCP8.5 with $\mathrm{CN}$ ) model configurations (so-called compsets). For simulations with prescribed SSTs we use the F_1850-2000_CN (20th Century transient stand-alone cam default, prescribed ocean/ice, with CN) compset (without modifications until 2005, with future atmospheric composition from the B_RCP8.5_ $\mathrm{CN}$ compset afterward). Simulations with prescribed SM and/or SSTs are started with initial conditions from the respective ensemble member of the reference simulation in 1996 and 2091. For the remainder of this study, we focus on two time periods: present day (1996-2005) and end of century (2091-2100).

\section{b. Experimental design}

To assess the influence of SM and SST dynamics on global land climate, we perform three different experiments (see Table 1) in the present-day and end-ofcentury time periods: (i) the above-described reference simulations with fully interactive land and ocean model components (REF); (ii) an experiment with removed interannual soil water variance, where we prescribe SM (across all soil levels) and soil ice at each grid point to the median value of the respective day of the year, as inferred from REF (noSMvar; note that this procedure allows us to preserve a seasonal cycle); and, similarly, (iii) an experiment with removed interannual SST and sea ice variance (noOCNvar). The noOCNvar experiment is performed with prescribed median values of the respective day of the year. Median values are calculated from 10 (years per ensemble member: 1996-2005 or $2091-2100) \times 10$ (ensemble members $)=100$ years. To test the sensitivity of this setup to the prescribed SST/sea ice, we additionally compute the experiment noOCNvar_neutral in which SST and sea ice are prescribed to values from the most ENSO-neutral year in REF. We compute the most ENSO-neutral year as the year with the lowest sum of the (absolute) monthly SST anomalies in the Niño-3.4 region. This region extends 
between $5^{\circ} \mathrm{S}$ to $5^{\circ} \mathrm{N}$ and $170^{\circ}$ to $120^{\circ} \mathrm{W}$. Note that even if this study focuses on the impacts of SST and SM on climate, for consistency we also prescribe sea ice and soil ice in the fixed SST and fixed SM simulations, respectively. Also, we prescribe in this study medians instead of means, which were generally prescribed to remove variability in previous studies (e.g., Seneviratne et al. 2006a; Jaeger and Seneviratne 2011; Seneviratne et al. 2013; Douville et al. 2016). Taking the mean introduces soil ice and sea ice as soon as it is present in a single ensemble member. Consequently, melting and albedo effects are overestimated, with potential impacts on the large-scale circulation (M. Hauser 2016, personal communication). Using the median therefore allows us to prescribe a representative duration of the presence of soil ice and sea ice. However, the soil ice and sea ice treatment should be of minor importance for our analysis, as we focus on the warm season and do not consider high latitudes (see section 2c).

To investigate the possible additivity of SM- and SST-related effects on climate, we additionally perform an experiment removing both interannual soil water and SST/sea ice variance (noALLvar). Moreover, to exclude impacts of removed SST/sea ice variance on soil water and vice versa, we compute two additional experiments, noSMvar* and noOCNvar*: in these experiments, we prescribe the SST and SM time series from REF, respectively, instead of calculating SST/sea ice and soil water interactively. An overview of all model experiments is provided in Table 1. Note that the focus in this study is on the REF, noSMvar, and noOCNvar experiments highlighted in bold in Table 1 . The other experiments are only needed for targeted additional analyses.

As we are investigating transient climate simulations with slowly changing atmospheric composition in this study, we assess trends in SM, SSTs, and 2-m land temperature in REF to exclude potential impacts on our results. Trends are computed at each location over the present-day period (10 yr) using mean fields across the 10 ensemble members for each month (warm-season months in the case of SM and land temperature, all months in the case of SSTs). We find significant trends at the $10 \%$ level in $15 \%, 9 \%$, and $11 \%$ of all grid cells for SM, SSTs, and land temperature, respectively. Similar results $(11 \%, 3 \%$, and $13 \%$ of the grid cells display significant trends, respectively) are obtained when considering only the first 3 years. At this significance level, significant trends are expected by chance in $10 \%$ of the grid cells; hence, this indicates that there are no profound trends in key variables of REF.

\section{c. Analysis of experiments}

From each of the experiments (REF, noSMvar, noOCNvar, and additional experiments; see Table 1), there are 10 (ensemble members) $\times 10$ (years per time period) $=100$ years of data for the present-day period and for the future period. With these data, we investigate changes in temperature, precipitation, and droughts following the removal of interannual SM or SST variability. For this purpose, we assess changes in means and variabilities between the experiments. Whereas we consider all data for the temperature analysis, the precipitation analysis focuses on events exceeding $1 \mathrm{~mm} \mathrm{day}^{-1}$, and for the drought analysis we extract the maximum number of consecutive dry days within the warm season at each location. The results are averaged from all land points in three different latitudinal bands (Fig. 1): tropics $\left(20^{\circ} \mathrm{N}-\right.$ $\left.20^{\circ} \mathrm{S}\right)$, subtropics $\left(20^{\circ}-35^{\circ} \mathrm{N}, 20^{\circ}-35^{\circ} \mathrm{S}\right)$, and midlatitudes $\left(35^{\circ}-65^{\circ} \mathrm{N}, 35^{\circ}-65^{\circ} \mathrm{S}\right)$. Furthermore, we only consider the warm season at each grid cell in our analyses: that is, the warmest three consecutive months; then the landatmosphere coupling is generally stronger, and we can exclude (sea or land) ice-related impacts on our results. The latitudinal regions along with the local warm seasons (as determined from REF) are shown in Fig. 1. In an additional analysis we use the boreal summer (JuneAugust) instead of the location-dependent warm season to test the role of the chosen season (see section 3d).

For the analysis of variabilities, we focus on anomalies to exclude the impact of (changes in) the seasonal cycle. Anomalies are derived by subtracting the long-term mean of each day of the year at each grid cell using the total 100 years of data for each experiment. We compute variabilities on three different time scales: day to day, month to month, and year to year. To ensure that the sum of these particular variances equals the total variance, we proceed as follows: We first compute yearly means (or actually 3-monthly means, as we focus on the warm season) and then the variance thereof (year-to-year variance). Afterward, we subtract the yearly means from the time series and compute monthly means and the variance thereof (monthto-month variance). Finally, we subtract also the monthly means and compute the variance of the resulting time series (day-to-day variance). This procedure is illustrated in Fig. 2. Note that we cannot perform the variability analysis for droughts, as there is only one estimate per season.

In addition to the overall changes in means and variabilities we compute changes in decile ranges (0-10, $10-20, \ldots$, and $90-100)$ of the considered variables at each grid cell. This allows us to analyze if changes differ between cold/warm temperatures, weak/strong precipitation events, or short/long droughts. Whereas the overall change in the means of temperature, precipitation, and droughts equals the mean of the decile changes, this is not the case for the variability changes. This is because the variance is based on squared differences with respect to either the mean of the decile range or the overall mean. 

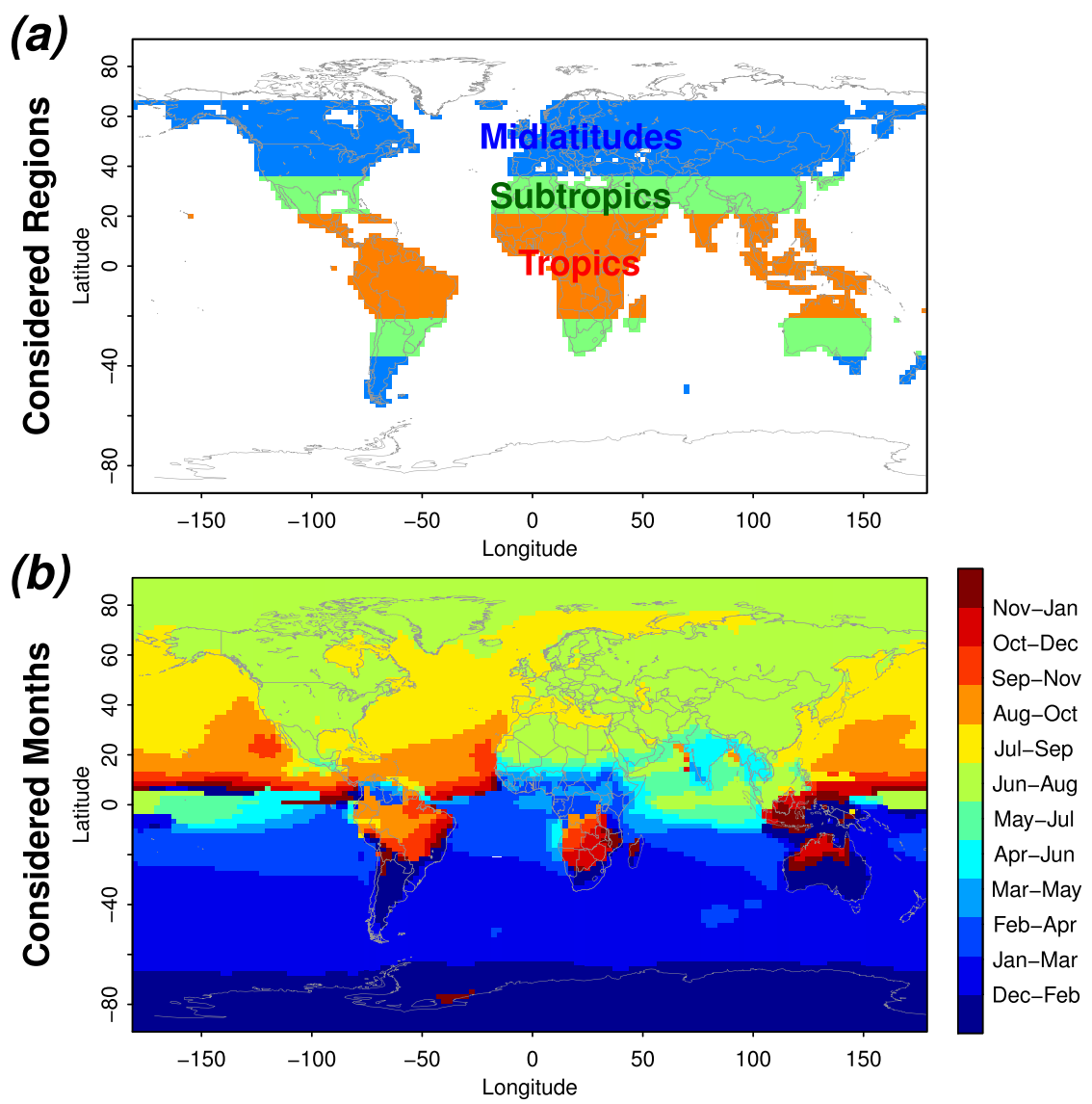

FIG. 1. (a) Considered latitudinal regions: tropics $\left(20^{\circ} \mathrm{N}-20^{\circ} \mathrm{S}\right)$, subtropics $\left(20^{\circ}-35^{\circ} \mathrm{N}\right.$, $\left.20^{\circ}-35^{\circ} \mathrm{S}\right)$, and midlatitudes $\left(35^{\circ}-65^{\circ} \mathrm{N}, 35^{\circ}-65^{\circ} \mathrm{S}\right)$. (b) Considered warm-season months at each grid cell.

Besides comparing changes in land climate means and variabilities in response to removed SM or SST variability, we additionally assess the roles of SM and SSTs for the temporal variability of land climate in a correlation analysis. We correlate monthly temperature and precipitation from REF and noSMvar* with identical SSTs to estimate the SM impact and from REF and noOCNvar* with identical SM to estimate the SST impact.

\section{SIGNIFICANCE TESTING}

After computing changes in land climate due to removed SM and SST variability, respectively, we also assess their significance. Significance assessment for all analyses in this study is performed with a bootstrapping approach. At each grid cell and for each experiment, we perform a random resampling of warm seasons with replacement to yield time series of temperature, precipitation, or cumulative dry days of the same length as the original time series, and with the same seasonal cycle. This is repeated 200 times to recompute each analysis 200 times with the respective resampled time series (e.g., to yield
200 estimates of the differences in means and variabilities of the considered variables between the considered experiments). Based on these 200 estimates, whiskers in box plots display the range between the $5 \%$ and $95 \%$ quantiles. Differences between experiments shown in maps are considered significant if the sign is the same in at least $95 \%$ (=190) of the performed resamplings.

We apply a $t$ test to assess the significance of correlations computed throughout this study, in which we also use a $95 \%$ confidence threshold to determine if correlations are significantly different from zero.

\section{Results}

\section{a. Climate means}

To infer the impacts of SM and SST variability on mean land climate, we compute mean differences between noSMvar and REF, and noOCNvar and REF, respectively. We focus here and in the next subsection on the present-day time period 1996-2005. Figure 3 provides global maps of mean climate changes. The 


\section{Example: Temperature, Central Europe}
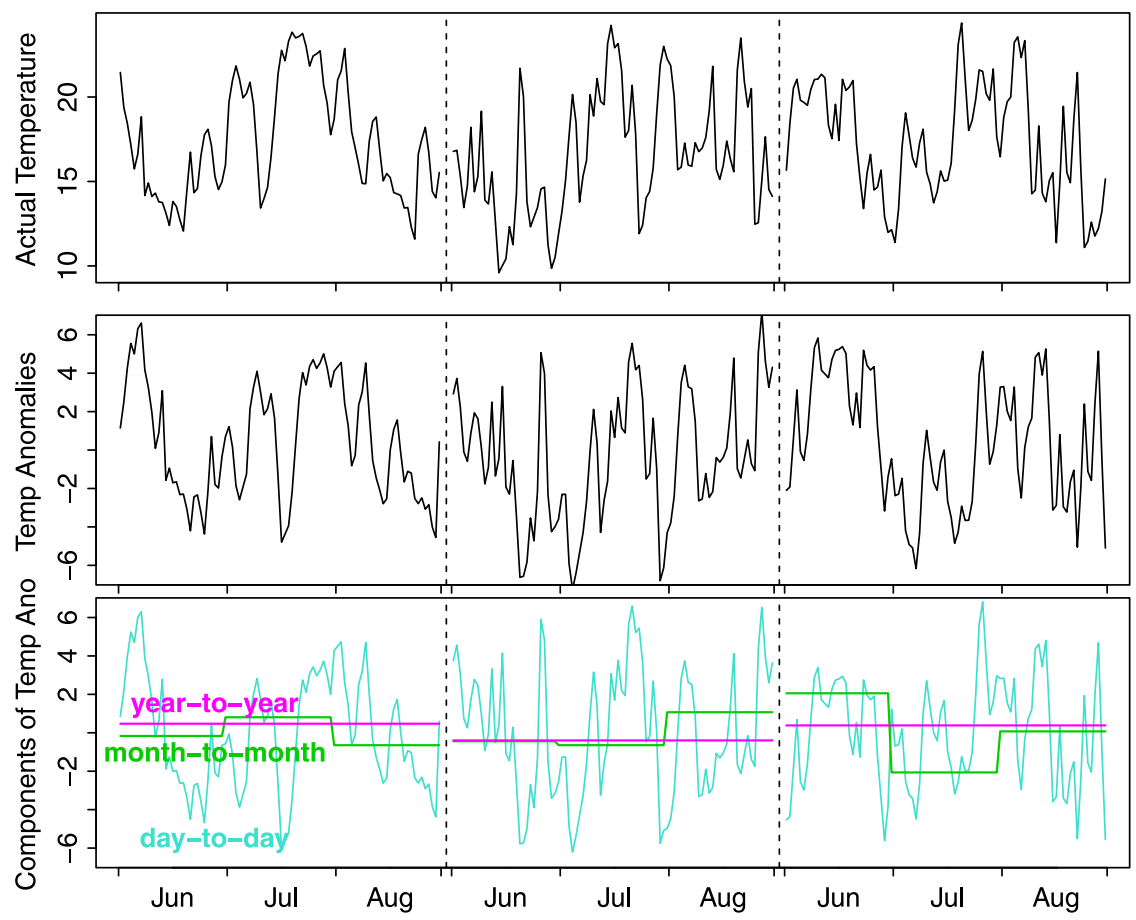

FIG. 2. Example of decomposition of an (anomaly) time series into seasonal, monthly, and daily components. These are then used to decompose the total variability into year-to-year, month-to-month, and day-to-day variability.

removal of SM variability causes stronger modifications in mean land climate than the removal of the SST variability. We find an overall cooling in both experiments. In the case of the noSMvar experiment, the pattern of negative temperature anomalies resembles the known pattern of present-day land-atmosphere coupling hot spots, except for the Sahel region and India [determined from covariability of land and atmospheric variables (Koster et al. 2004; Seneviratne et al. 2006a; Guo et al. 2006; Dirmeyer 2011; Mueller and Seneviratne 2012)], whereas it is more uniform in the noOCNvar experiment. The cooling in response to the removed SM variability can be explained with the shape of the relationship of evaporative fraction (EF; evaporation scaled by net radiation) and SM: if soils are wet, EF is largely independent of SM, whereas, if soils are drier, plants start to conserve water and bare soil evaporation decreases such that EF becomes linearly dependent on SM [see, e.g., Seneviratne et al. (2010, Fig. 5 therein)]. When SM is fixed to the median seasonal cycle, there are an equal number of wet days getting drier and dry days getting wetter. The former will have less (or no) impact on $\mathrm{EF}$ in contrast to the latter such that the overall EF increases. The higher evapotranspiration in regions with strong land-atmosphere coupling is displayed in Fig. 4.
The figure also suggests that the increased evapotranspiration induces the surface cooling (i) through consequently decreased sensible heat flux, and (ii) because it contributes to increased cloud cover and consequently decreased surface radiation. Furthermore, wind speed is reduced in regions with increased evapotranspiration, which might be due to increased vegetation cover in response to the removal of dry SM extremes. In contrast to this cooling we find a warming across dry areas, such as the Sahara, the Arabian Peninsula, and western North America [similar to results in Berg et al. (2014)]. This can be explained with lower soil moisture shown in Fig. S1, which then leads to warmer temperatures through the same mechanism as described above. Mean soil moisture in these areas is reduced by prescribing the median values because the median is drier than the mean as a result of a skewed soil moisture distribution with many dry days and a few (very) wet days, which occur after rain events. The cooling we observe in the noOCNvar experiment might be related to the fact that the spatial pattern of the prescribed median SSTs is not necessarily exactly ENSO neutral (see also section 3d). In both experiments, precipitation changes are rather patchy and tend to be insignificant outside the tropics, and correspondingly there are almost no significant 


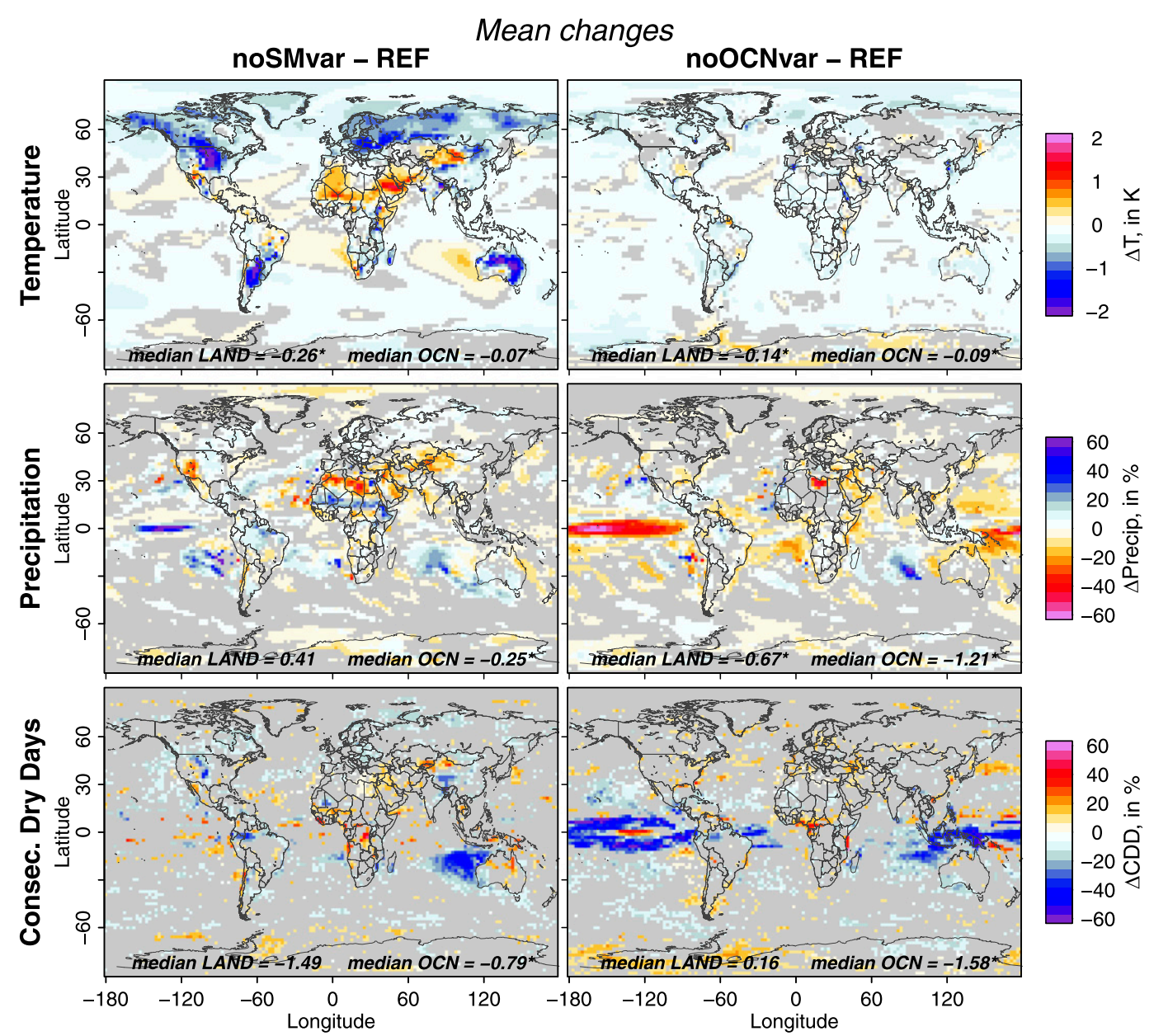

FIG. 3. Maps of impacts of removed (left) SM and (right) SST variability on the means of (top) temperature, (middle) precipitation, and (bottom) consecutive dry days. For precipitation and consecutive dry days, impacts are expressed as relative changes. Gray areas denote insignificant changes at the 5\% level (see text for details). The significance of median estimates in each plot is denoted by a star (at the $5 \%$ level).

changes in drought over land, only some reduction in the cumulative dry days over tropical oceans. Interestingly, in the noSMvar experiment we find opposite mean precipitation changes in the Sahel as compared with other desert areas even though such a spatial pattern is not observed in any other variable considered in Fig. 4. Removed SST variability causes precipitation changes that resemble a (reversed) ENSO-related pattern (Dai and Wigley 2000). In particular, we find a strong reduction of precipitation in the noOCNvar experiment in the eastern equatorial Pacific, which is accompanied by fewer clouds and more radiation. Like the temperature changes in the noSMvar experiment, these changes might be related to a nonlinear response of precipitation to ENSO (Chung et al. 2014).

Figure S2 analyzes potential controls of changes in temperature, precipitation, and drought in order to help understanding the described spatial patterns of land climate change. The scatterplots roughly confirm the role of local precipitation coupling with ENSO for precipitation changes in the noOCNvar experiment in the tropics, whereas there is no relationship in other regions and in the case of temperature and drought changes. The low explanatory power of the climate-ENSO coupling might be related to the fact that there are other controls involved. In the noSMvar experiment, there is an influence of land-atmosphere coupling and SM variability on temperature changes, which is in line with the SMinduced changes in surface energy fluxes discussed above. For precipitation and droughts, the relationship is weaker, with only a tendency toward larger (positive and negative) changes in case of a positive landatmosphere coupling. This behavior is found in all latitudinal regions. However, also, in the case of the noSMvar experiment, the tested controls cannot fully explain the observed climate changes. 


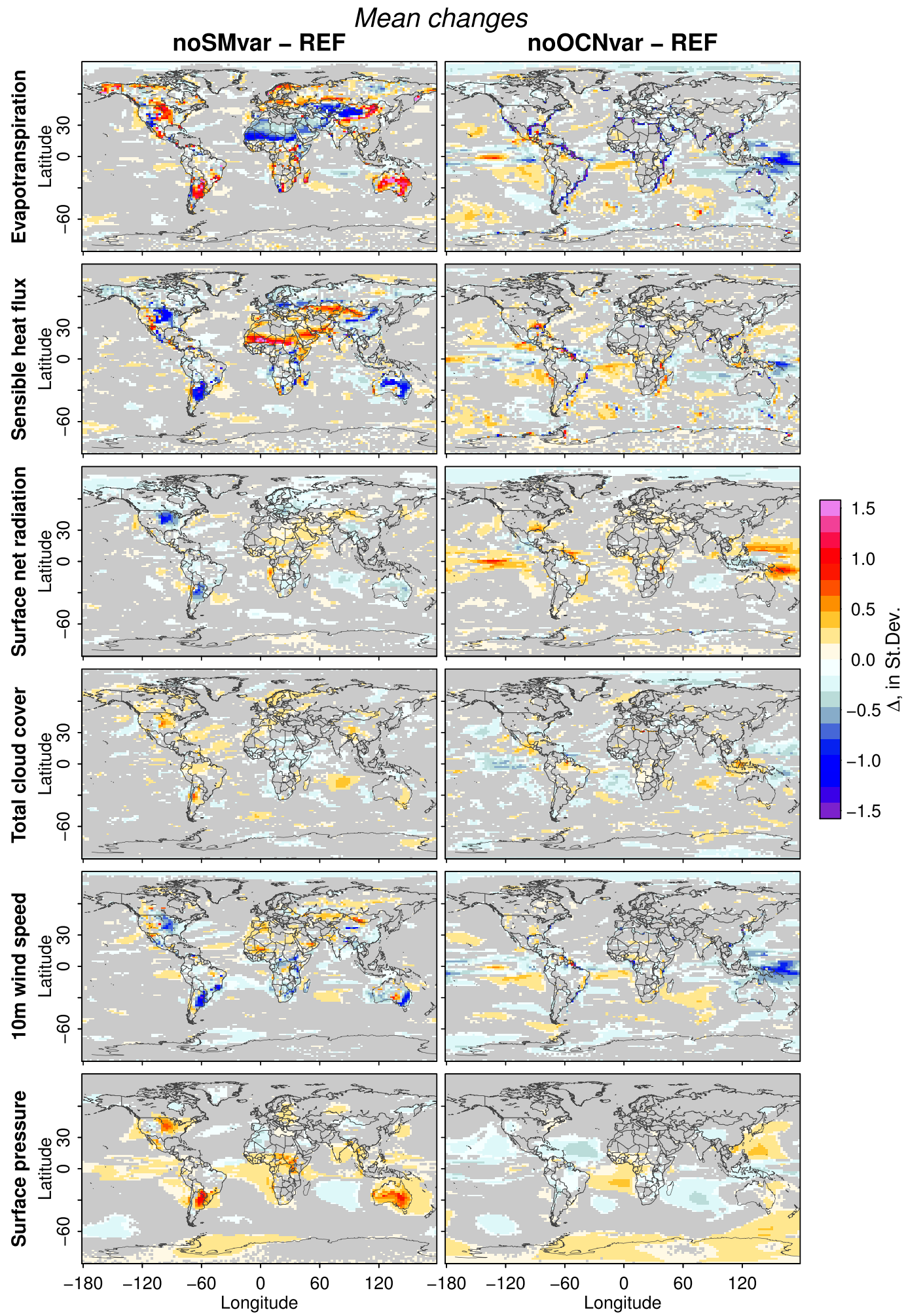

FIG. 4. As in Fig. 3, but for mean changes in surface fluxes, radiation, cloud cover, wind speed, and pressures. All changes are expressed in standard deviations of the respective variability in REF. 


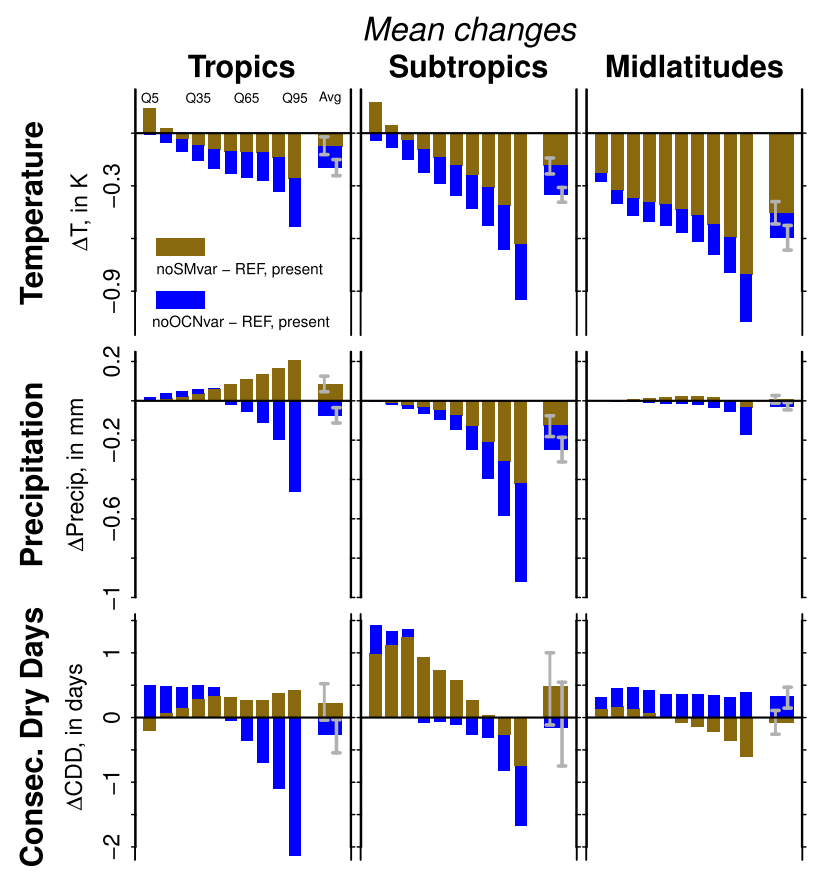

FIG. 5. Impacts of removed SM (brown) and SST (blue) variability, respectively, on mean (top) temperature, (middle) precipitation, and (bottom) consecutive dry days, averaged over latitudinal regions (Fig. 1) in the respective warm seasons at each grid cell. Results are shown for the present-day period (1996-2005). The wider bars to the right in each plot refer to average changes, whereas the other bars illustrate changes in different deciles. Whiskers indicate the $5 \%-95 \%$ confidence range.

Averaging the described climate differences across latitudinal land regions, Fig. 5 displays the results for temperature, precipitation, and cumulative dry days. SM and SST variability, respectively, have overall a similar influence on mean land climate in the CESM experiments; SM is even found to be more important in the midlatitudes. The similar importance of SM versus SSTs for land climate means is a main result of this study. Generally mean temperature differences are largest in the midlatitudes, whereas precipitation and drought changes are strongest in the tropics and subtropics. Analyzing the decile changes, we find a strong asymmetry of SM-induced temperature changes with strongest impacts on hot temperatures. This can be explained by the asymmetric relationship between SM and $\mathrm{EF}$, which is stronger toward drier conditions. As these conditions are usually associated with hot temperatures, the warm end of the temperature distribution is most strongly impacted by the removal of SM variability. This signature of the SM-temperature feedback was also reported in the GLACE-CMIP5 experiments (Seneviratne et al. 2013). Precipitation changes are small but significant, in contrast to the drought changes, which are mostly insignificant. The comparatively small precipitation changes may be because precipitation formation involves several processes acting within the higher atmosphere, whereas temperature is more strongly controlled by surface fluxes. Furthermore, models with parameterized convection schemes, such as CESM, tend to be less sensitive to the surface fluxes than in the case of models with explicitly resolved convection (P. Dirmeyer 2016, personal communication).

\section{b. Climate variability}

In addition to the impacts of SM versus SST on the mean state of land climate, we have also investigated the impacts on variability. For a more comprehensive analysis, we split up the variability changes into three time scales: day to day, month to month, and year to year, as described in section 2c. Changes in temperature and precipitation variability are shown in Fig. 6. As for the mean climate changes, we find generally a similar importance of SM and SST variability in the warm season; there is, moreover, again a tendency toward larger changes at higher deciles. Note that the mean of the decile variability changes may differ from the overall variability change because the variance is based on squared differences with respect to either the mean of the decile range or the overall mean (see section $2 \mathrm{c}$ ). Both temperature and precipitation variability are more impacted at lower latitudes and longer time scales. Interestingly, the relative reductions of climate variability in the noSMvar and noOCNvar experiments depend on the considered time scale; while climate variability at the daily time scale is much more strongly reduced in noSMvar compared to noOCNvar, the reductions are similar to or even slightly larger in the case of the noOCNvar experiment at the interannual time scale. Temperature variability changes are comparatively large, ranging up to $30 \%-40 \%$ for removed SM or SST variability, respectively, whereas precipitation variability changes are mostly below $10 \%$. Interannual variability of hot tropical temperatures seems to be almost entirely governed by SM and SST variability in CESM. Corresponding to removed variability of SM and SSTs, respectively, all climate variability changes are also reductions, except for the tropical precipitation variability, where we find a small but significant increase in the noSMvar experiment. Note further that the variance may not be well suited to capture differences between heavy-tailed distributions, which are characteristic of precipitation. Therefore, we repeated the analysis using the interquartile range and found similar results (not shown).

As land climate variability changes were strongest at the interannual time scale in the case of temperature 


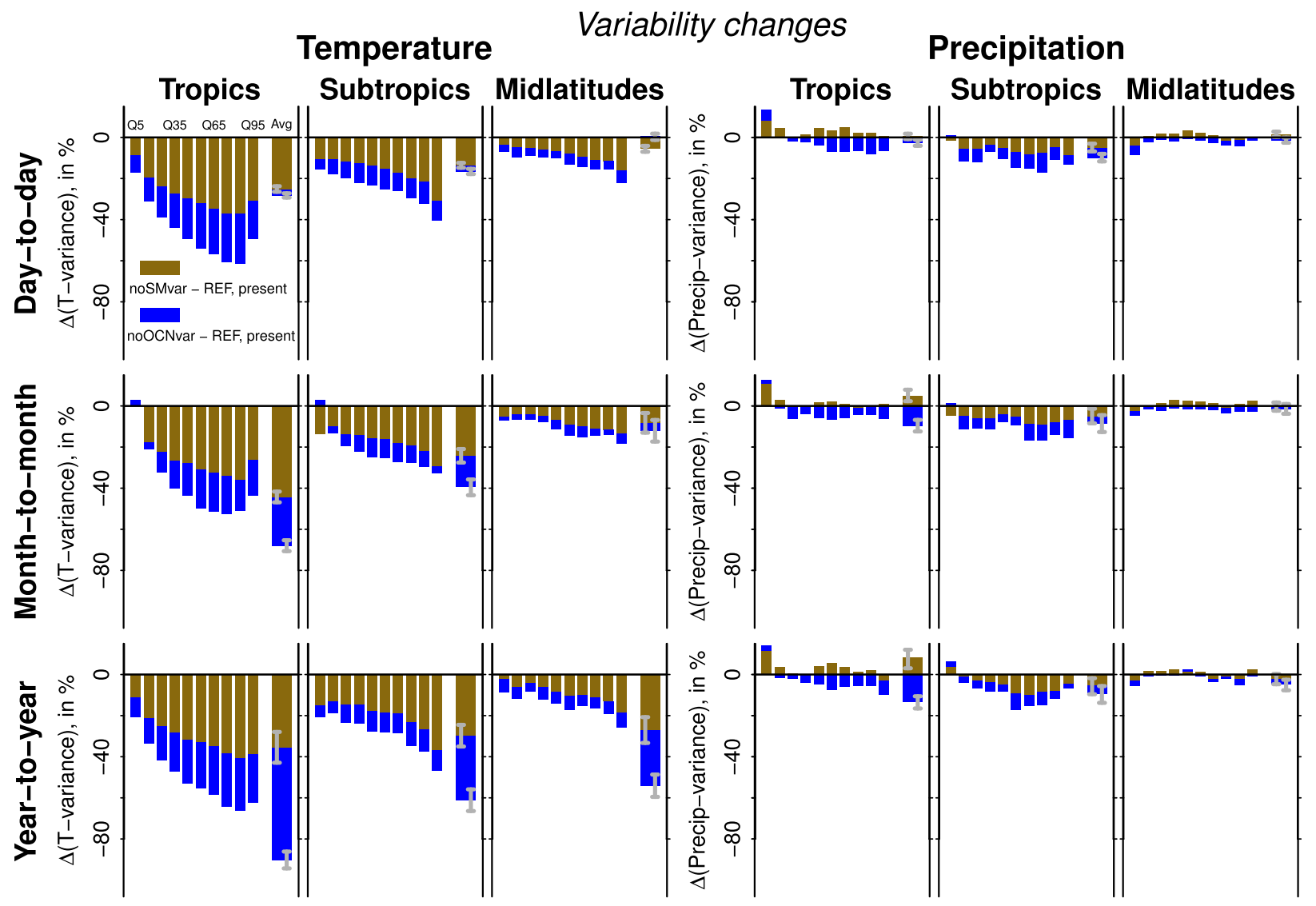

FIG. 6. As in Fig. 5, but for relative variability changes of temperature and precipitation as a response to removed variability in SM and SST, respectively. Each row refers to a different time scale of variability (see section $2 \mathrm{c}$ ). Whiskers indicate the $5 \%-95 \%$ confidence range.

and also comparatively strong in the case of precipitation, we focus on this time scale in the following. Corresponding global maps for temperature and precipitation are provided in Fig. 7 [similar to Figs. 2 and 4 in Dirmeyer (2001)]. The results resemble the patterns in Fig. 3: precipitation changes are mostly insignificant, except for the tropics. Especially when removing SM variability over land in the noSMvar experiment, precipitation variability over the ENSO region in the tropical Pacific Ocean is strongly reduced. Temperature variability changes in that experiment occur mostly in the hot spot regions of land-atmosphere coupling. These temperature variability changes are caused by strong changes in the variabilities of the surface fluxes, as shown in Fig. 8, which, however, only impact surface temperature variability in regions where it depends on EF. Interestingly, the removal of SST variability in most regions has no impact on precipitation variability, except for the tropical Pacific, where we find a strong reduction, accompanied by less variability in all other investigated variables. Naturally, the removal of SST variability strongly reduces the variability of 2-m temperature over the oceans, and the changes propagate inland especially in lower latitudes.

Figure S3 displays the analysis of the temperature and precipitation variability changes with respect to the correlation of local temperature or precipitation with the Niño-3.4 index in the case of the noOCNvar experiment, and against the land-atmosphere coupling strength and the undisturbed SM variance in the case of the noSMvar experiment (as for the mean changes in Fig. S2). Consistent with the fact that mean and variability changes display similar patterns, we also find similar results for the control mechanisms (ENSO and SM-ET coupling). In contrast to precipitation variability changes, temperature variability changes in the noSMvar experiment can be explained to some extent with the strength of the SM-ET coupling in REF: Because ET is connected with sensible heat flux and hence temperature through the surface energy balance, SM can influence surface temperature in the case of a strong SM-ET coupling. This is also illustrated with the strongly reduced variabilities of the surface energy 


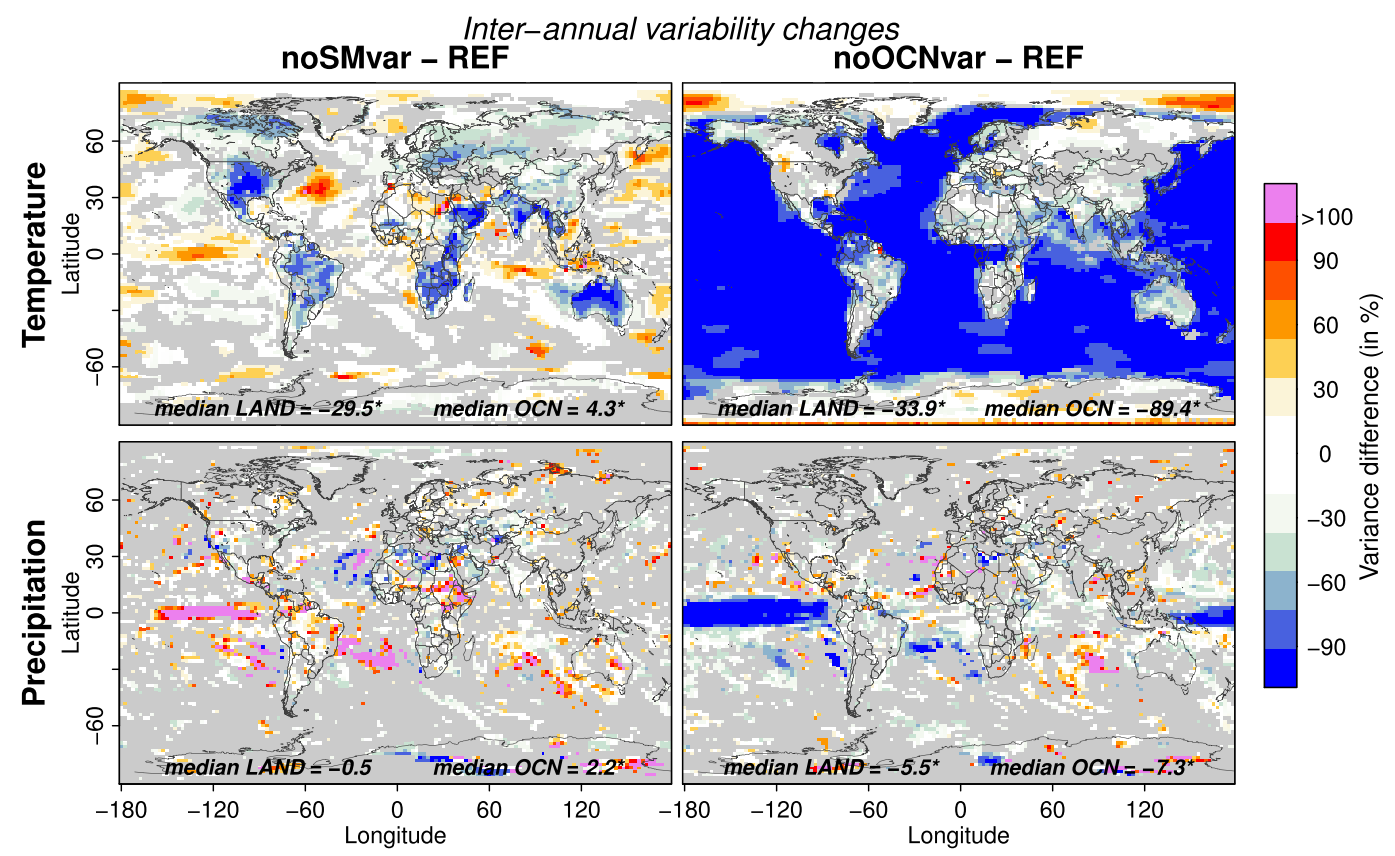

FIG. 7. Maps of changes in year-to-year variability of (top) temperature and (bottom) precipitation as a response to removed variability of (left) SM and (right) SST. Changes are expressed relative to the respective total variability in REF. Gray areas denote insignificant changes at the 5\% level. The significance of median estimates in each plot is denoted by a star (at the $5 \%$ level).

fluxes in Fig. 8. Hence, the removal of SM variability reduces temperature variability more strongly in the case of a strong SM-ET coupling and/or high natural SM variability. In the noOCNvar experiment, only changes of tropical precipitation variance are related to the precipitation-ENSO coupling; no relationship is found in the other regions and in the case of temperature variability. However, the latter seem to be limited by a strong temperature-ENSO coupling. Variability changes in the noSMvar experiment are controlled by land-atmosphere coupling in all latitudinal regions. The link is clearly stronger for temperature than for precipitation. Temperature changes are furthermore related to SM variability in REF. Note that, as in the case of the mean climate changes, the tested controls cannot fully explain the observed changes in climate variability, indicating that other controls are involved that are not considered here.

\section{c. Nonlocal effects of soil moisture}

In Figs. 3, 4, 7, and 8, there are cases where removed SM variability on land introduces some climate changes over the oceans. In addition, there can also be nonlocal effects from SM on land, whereby SM anomalies affect climate in another location, either through triggering circulation changes or by impacting heat and moisture in local near-surface air masses, which are then advected to another location. In this section, we discuss these nonlocal effects of SM in more detail. (It goes without saying that all effects from SST on land climate are nonlocal, and these can be well assessed from the above-listed figures.)

We note that the nonlocal SM effects can only partly be assessed: (i) nonlocal effects of SM on ocean climate can be assessed and are found to be weak and only significant in few regions (see above-listed figures); (ii) on the other hand, while much of the SM effects on land are expected to be local, nonlocal effects are possible (e.g., Seneviratne et al. 2013; Koster et al. 2014) but cannot be easily disentangled with our present modeling framework. Some of the nonlocal effects are caused by impacts of local SM or SST changes on the large-scale circulation, which then lead to changes in the advection of air masses and finally to precipitation or temperature changes in places located (far) away from the local SM or SST changes (e.g., Hoskins and Karoly 1981; Koster et al. 2014). In addition, other nonlocal effects without changes in circulation patterns can be induced as SM impacts temperature and moisture in local air masses, which can then be advected to another location.

In the following, we analyze the impact of the SM and SST variability on circulation patterns, to assess one source of nonlocal effects. These corresponding 


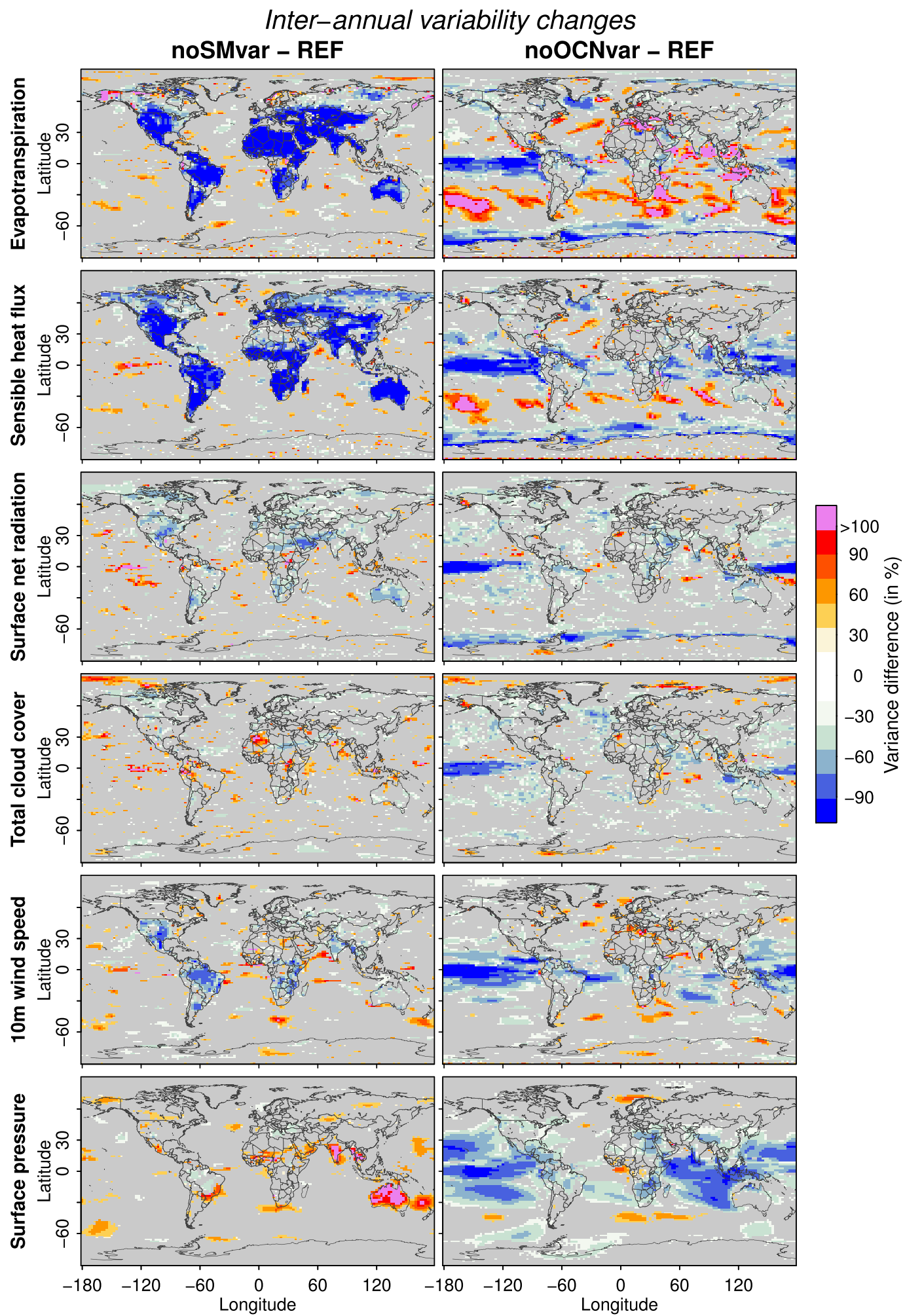

FIG. 8. As in Fig. 7, but for variability changes in surface fluxes, radiation, cloud cover, wind speed, and pressures. 

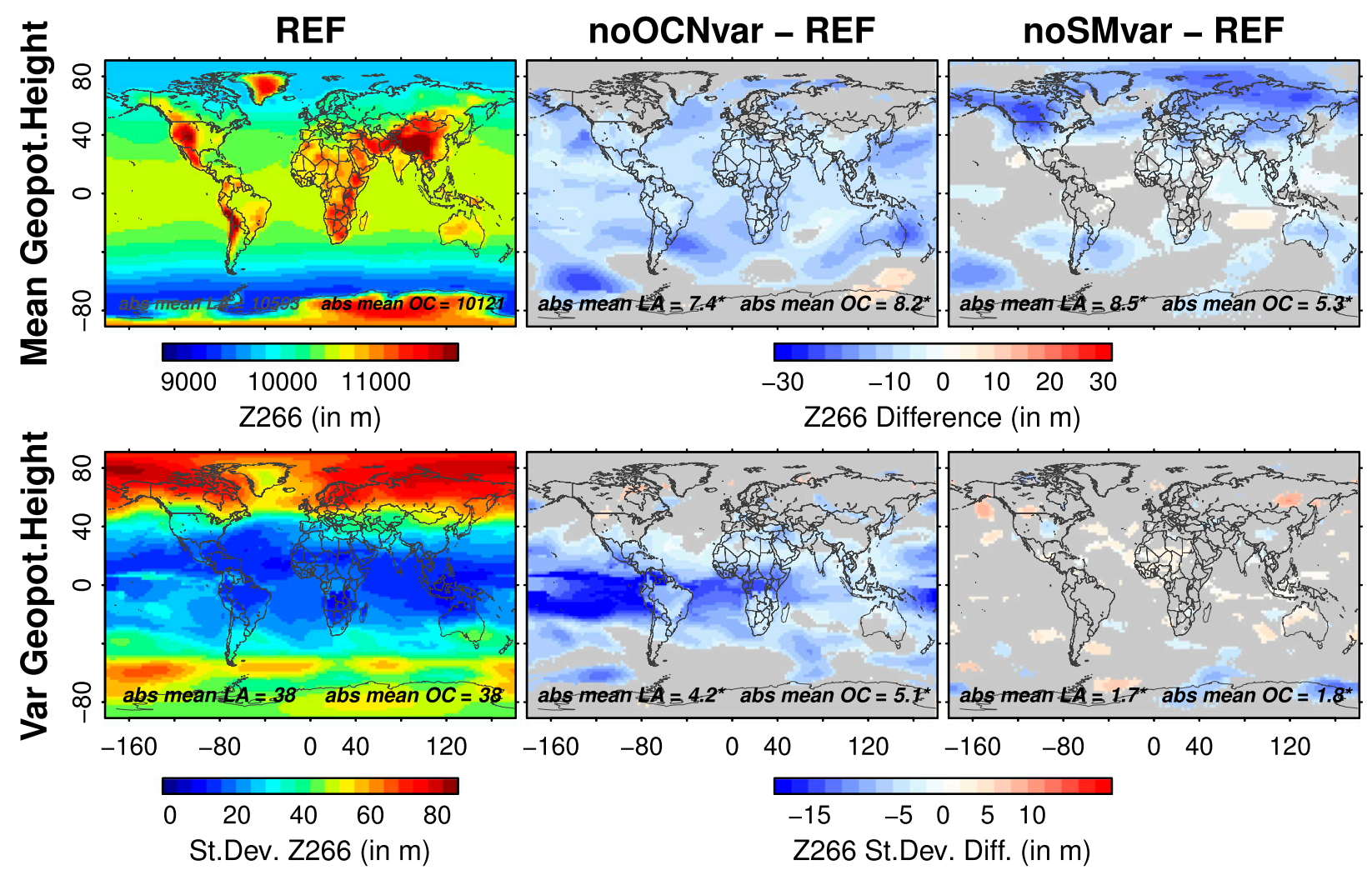

FIG. 9. Impacts of removed SM and SST variability, respectively, on geopotential height at a height of 266 mbar. Results are displayed for changes in mean and variability. The significance of mean estimates in each plot is denoted by a star (at the $5 \%$ level).

large-scale circulation changes are illustrated in Fig. 9, where we chose the geopotential height as an indicator of large-scale upper-atmosphere dynamics. We investigate changes in geopotential height at $266 \mathrm{mbar}$ (or at sigma $=0.266$; CESM includes hybrid sigma-pressure levels). Even though the SM or SST changes we apply are intrinsically limited to land or ocean, respectively, there are changes in geopotential height throughout the globe. These changes reflect variations in the largescale atmospheric circulation that lead to changes in, for example, surface pressure and wind over some ocean regions in the case of removed SM variability and over some land regions in the case of removed SST variability, respectively (see Figs. 4, 8). These changes consequently translate into nonlocal impacts on surface climate. This involves a chain of complex processes that we do not further consider here. Changes are field significant (significant in more than $5 \%$ of the area) in all latitudinal regions in both analyses.

Nonlocal induced impacts on climate comprise variations in (i) means and (ii) variability. In the case of variations in mean climate, nonlocal effects are, for example, responsible for the significant temperature changes over the oceans in the noSMvar experiment. In the case of variations in climate variability, these effects could play a role for the increased variability in multiple climate variables across the eastern equatorial Pacific Ocean in the noSMvar experiment (Figs. 7, 8). [Note that the latter two examples are rare cases where we find significant SM-induced effects on the oceans, while SST-induced effects on land (i.e., land climate changes in the noOCNvar experiment) are obviously found in all analyses in this study.]

The existence of nonlocal SM effects implies that "direct" climate responses to modified SM at a particular location are the result of local SM effects, as well as of nonlocal changes introduced by SM changes in other regions. In this context, the nonlocal effects of SM could play a role in masking the underlying controls of the climate responses tested in Figs. S2 and S3, and they could probably contribute to the slightly asymmetric temperature response in the noOCNvar experiment. The influences of SST changes and SM changes on one another are shown in Figs. S1 and S4.

\section{d. Testing the role of climate change, of the prescribed SSTs, and of the selected season}

Figure 10 summarizes the previously described changes in land climate mean and variability. It furthermore displays the respective changes computed for 

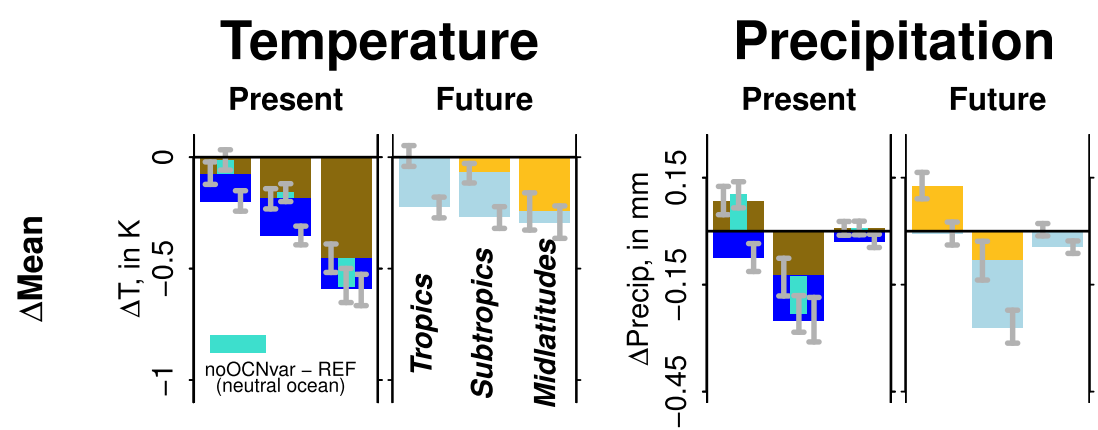

\section{Consec. Dry Days \\ Present Future}
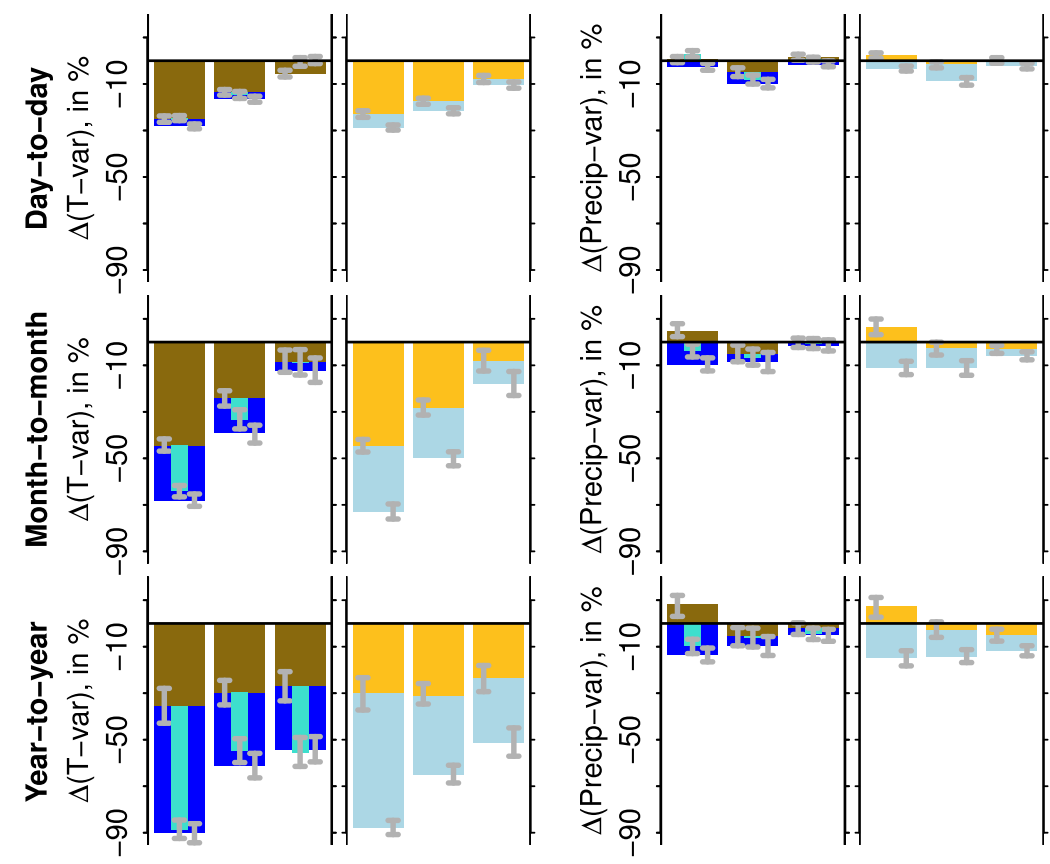

FIG. 10. Summary of average changes from Figs. 5 and 6 [for the present-day period 1996-2005 (brown/blue)] compared with respective results for the future period [2091-2100 (yellow/light blue)]. The turquoise bars illustrate the impact of the choice of the prescribed SSTs. As the blue bars, they show changes after removing SST variability, but with prescribed neutral SSTs rather than median SSTs (see section 2b). Whiskers indicate the $5 \%-95 \%$ confidence range.

the end of the century. This analysis shows that the results are similar. Only changes in mean temperature in the noSMvar experiment are significantly smaller in the future, likely because of a weaker SM-EF coupling in CESM in a warmer world. Note that the latter is in contrast to results from Dirmeyer et al. (2013a), where the multimodel average indicates a stronger SM-EF coupling in a changing climate. Nevertheless, given that almost all other differences between the results for present and future climate are insignificant (overlapping confidence intervals of the computed estimates), our conclusion that SM and SST variability are similarly important for land climate in the warm season is independent of global climate change.

In the experiments for the present-day period we also test the role of the choice of the prescribed SSTs in
noOCNvar. The turquoise bars in Fig. 10 show the results with prescribed neutral SSTs instead of median SSTs (see section 2b). In terms of the variability changes, we find similar results (i.e., no influence of the chosen SSTs). In contrast, significant changes in land climate means are found indicating sensitivity of the mean climate to the prescribed SSTs.

Furthermore, we test the influence of the locationdependent warm season on our results by additionally performing the analyses during boreal summer (see section 2c). The corresponding results shown in Fig. S5 are very similar to previous results in Fig. 10 suggesting a minor role of the chosen warm season for our results. Strongest changes are found in the tropics, which is expected as the warm season in this region differs clearly from the boreal summer (see Fig. 1); 


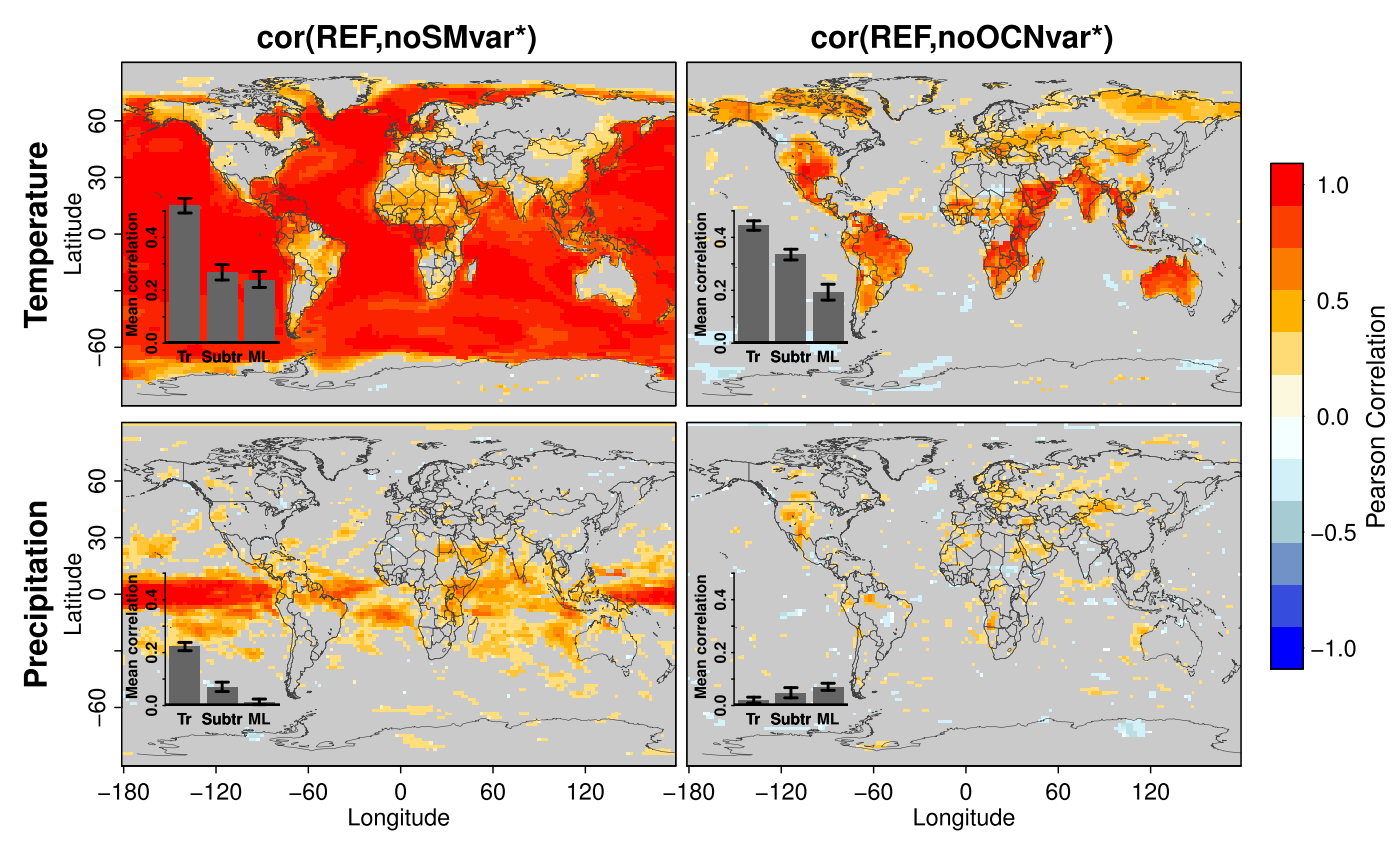

FIG. 11. Correlation of monthly mean temperature and precipitation (left) in the case of identical SSTs and different SM and (right) in the case of different SSTs and identical SM. Gray areas denote insignificant changes at the $5 \%$ level. Barplots denote mean correlations across latitudinal regions; whiskers therein indicate the $5 \%-95 \%$ confidence range.

however, they are mostly insignificant except for decreased tropical temperature mean and variability in the noSMvar experiment.

\section{e. Correlation analysis}

In addition to the assessment of SM and SST impacts on land climate means and variabilities, we moreover perform a correlation analysis to infer the influence of removed SM or SST variability on the temporal dynamics (i.e., changes over time) of land climate (see section 2c). For this purpose, we compare REF with noSMvar* and noOCNvar*, as they share the same SSTs and SM, respectively. This allows us to isolate the impact of removed SM and SST variability, respectively, on the temporal evolution of land climate. The results are displayed in Fig. 11 Temperature dynamics are overall better preserved than precipitation dynamics when using identical SM or SST conditions, as shown by the higher correlations, and climate dynamics at lower latitudes are better preserved than at higher latitudes. Comparing the results with identical SSTs (left) and SM conditions (right), we find generally similar mean correlations across latitudinal bands over land, which confirms the finding from the previous subsections that the role of SM for land climate is comparable to that of SSTs. Nevertheless, there are regional differences: in the tropics, climate dynamics are significantly better preserved with identical SSTs than with identical SM, whereas identical SM allows for better preservation of temperature dynamics in the subtropics and precipitation dynamics in the midlatitudes.

\section{Discussion}

\section{a. Are SM and SST variability independent?}

In the previous sections, we separately analyzed the impacts of removed SM and SST variability on land climate. This involves the assumption that these two variabilities are independent such that, for example, the removal of SST variability would only impact land climate directly and not by inducing changes in SM variability. As shown in Figs. S1 and S4, this is not everywhere the case, even though in most regions SM acts independently of SST impacts. We perform an analysis to test this independence assumption in the following, similar to the additivity analysis in Koster et al. (2000).

If SM and SST impacts in the warm season are independent, the sum of the land climate changes from noSMvar and noOCNvar should equal the land climate changes in noALLvar. The corresponding analysis is presented in Fig. 12, where each point refers to a particular grid cell. We find significant relationships across all tested variables, as indicated by the significant correlations, as indicated by significant explained fractions of variance. A proof of perfect independence 


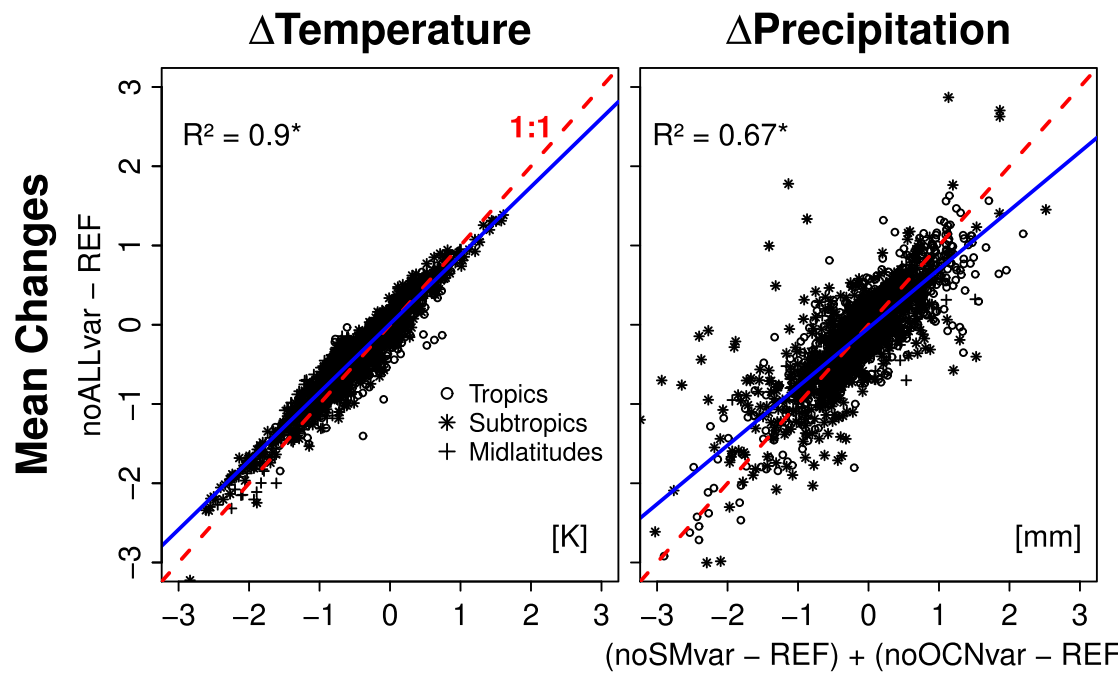

$\Delta$ Consec. Dry Days
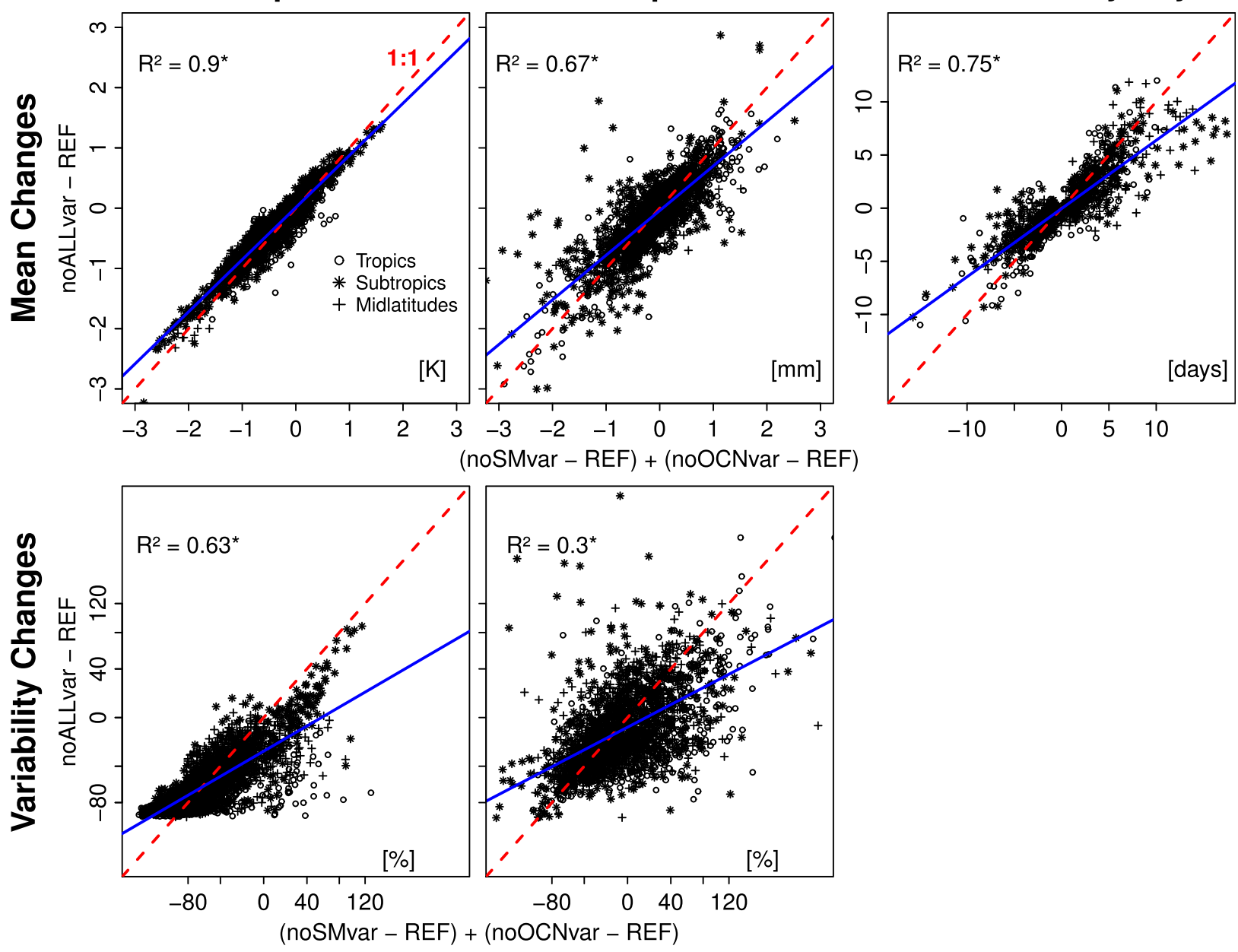

FIG. 12. Testing for similarity of added land climate changes in noSMvar and noOCNvar vs changes in noALLvar. Results displayed for significant changes in (top) means and (bottom) variabilities. Only land points are considered. Different symbols refer to different latitudinal regions. The significance of $R^{2}$ estimates is indicated by a star (at the $5 \%$ level).

additionally requires slopes close to 1 ; however, all slopes are significantly smaller than 1 , underlining that SM and SSTs are not fully independent. Generally stronger correlations are found for mean changes than for variability changes and in the case of temperature as compared to precipitation and drought. Weaker correlations and correspondingly smaller slopes are probably related to SM-induced nonlocal effects over land or "secondary" SM-induced nonlocal effects contributing to the observed climate changes (see section 3c) and thereby mitigating the independence of SM and SST-induced changes. Furthermore, weaker independence in the case of precipitation and drought changes could be related to the fact that these changes are smaller in relative terms compared with the size of the confidence interval (see Fig. 10). Overall, the effects are found to be larger when considered separately, which can be explained by additional SM changes induced by the removal of SST variability in the noOCNvar experiment (Fig. S1) as well as, to a more limited extent, by SST changes induced by the removal of SM variability in the noSMvar experiment. Furthermore some local amplification of SST-induced climate changes through land-atmosphere feedbacks could play a role. There are no systematic differences across the latitudinal regions.

To illustrate that the existence of the dependence between SST and SM changes does not impact the conclusions of our study, we recomputed the noSMvar and noOCNvar experiments with prescribed SSTs and SM from REF, respectively (see Table 1), thereby excluding impacts of SM variability on SST variability and vice versa. The resulting changes in land climate are illustrated and compared with previous results in Fig. S6. 
Overall, the results of the noSMvar* and noOCNvar* experiments are largely similar to results of noSMvar and noOCNvar; the differences in mean and variability changes are mostly within $\pm 10 \%$. Also, the role of SM for land climate in the warm season is comparable with that of SST. This confirms that the independence assumption is sufficiently fulfilled for the purpose of our study. One exception is the stronger mean cooling in the noOCNvar* experiment compared to noOCNvar. This is caused by the overall drier soil moisture conditions in the noOCNvar experiment, as shown in Figs. 3 and S1. Therefore, prescribing the soil moisture content from the REF experiment in the noOCNvar* experiment induces a wettening that leads to a cooling through the asymmetric SM-EF relationship (see also section 3a).

The overall sufficient degree of independence between SM and SST dynamics can be understood with the results in Fig. 6. In the CESM, SST variability affects precipitation variability over land only in some regions (see Fig. 11), and hence there is overall limited impact on SM (see Figs. S1 and S4). This contradicts the often-held assumption that SM mainly acts as a feedback to SST forcing. Nevertheless, this may occur in some regions, predominantly in the tropics. In contrast to our results, previous studies also found impacts of SSTs on precipitation variability in subtropical and midlatitude regions (Hoerling et al. 2006; Zhou et al. 2008; Bichet et al. 2011), with possible amplifications by SM feedbacks (e.g., Giannini et al. 2003; Schubert et al. 2004). On the other hand, we find some effects of SM on SSTs; however, these are generally small and not significant, as can also be inferred from the similarity between the noSMvar and noSMvar* temperature responses in Fig. S6.

\section{b. Is the employed Earth system model representative?}

While GCM experiments with fixed SSTs are common practice [first applied by Gates (1992)], there are few modeling experiments prescribing soil moisture alone, and a large range of model responses to prescribed SM has been shown in previous studies (Koster et al. 2004; Seneviratne et al. 2013). We test the robustness of our results in terms of the response to prescribed soil water content by comparing them with respective analyses of simulations from the GLACE-CMIP5 experiment (Seneviratne et al. 2013). Besides CESM, four additional GCMs participated in that experiment: L'Institute Pierre-Simon Laplace model (IPSL), the Geophysical Fluid Dynamics Laboratory model (GFDL), the ECEarth model (EC-Earth), and the Max Planck Institute Earth system model (ECHAM6). Using data from the present and future time periods (section 2a) and from all five GCMs that participated, we analyze the difference between two experiments: (i) the fully coupled reference simulations (similar to REF in this study) and (ii) simulations with SM prescribed to a transient climatology as computed with a $30-y r$ running mean from the respective reference simulation (referred to as experiment $\mathrm{B}$ in that study). In these simulations the interannual SM variability is removed (to a large extent), while possible trends in SM are preserved (similar to noSMvar* in this study, since SSTs are prescribed).

The differences between the two experiments with respect to land climate mean and variability are displayed in Fig. 13, along with respective results of this study. Generally, the changes computed in CESM and the other models are roughly comparable. In terms of climate variability changes, the CESM results are well within the range of the other models. Also mean precipitation changes in CESM are comparable, whereas the mean temperature decrease is slightly stronger than in the other models. The results obtained with CESM are overall comparable with those found with the other GCMs, and hence this confirms the representativeness of the CESM employed in this study to investigate the effect of removed SM variability. We can furthermore compare the CESM results from GLACE-CMIP5 with the results from this study. Note, however, that there are some methodological differences between the two studies: different considered time periods $(10-y r$ periods in this study with 10 ensemble members vs 30 -yr periods in the other with no ensemble), partly different considered seasons [warm season in this study versus June-August (Northern Hemisphere) and December-February (Southern Hemisphere) in GLACE-CMIP5], different SSTs (both interannually varying, but in this study taken from REF, in GLACE-CMIP5 from the respective CMIP5 simulations), different resolution $\left(2.5^{\circ} \times 2.5^{\circ}\right.$ in this study vs about $1^{\circ} \times 1^{\circ}$ in GLACE-CMIP5), different prescribed soil moisture climatologies (mean vs median soil moisture), and finally the soil moisture prescription that preserves long-term trends in GLACE-CMIP5 but not in this study. Nonetheless, we find large similarities between the CESM results in the two studies, especially in terms of land climate variability changes as a response to removed soil moisture variability, and also for mean precipitation changes. In contrast, the mean temperature change simulated by CESM is much stronger in the GLACE-CMIP5 study than in this study. This is perhaps due to the methodological differences outlined above. Finally, comparing the difference between the results of the noSMvar (interannually varying, interactive ocean) and noSMvar* (interannually varying ocean prescribed from REF) experiments of this study, we find that the differences caused by a (computationally very demanding) interactive ocean are small compared to 
Temperature
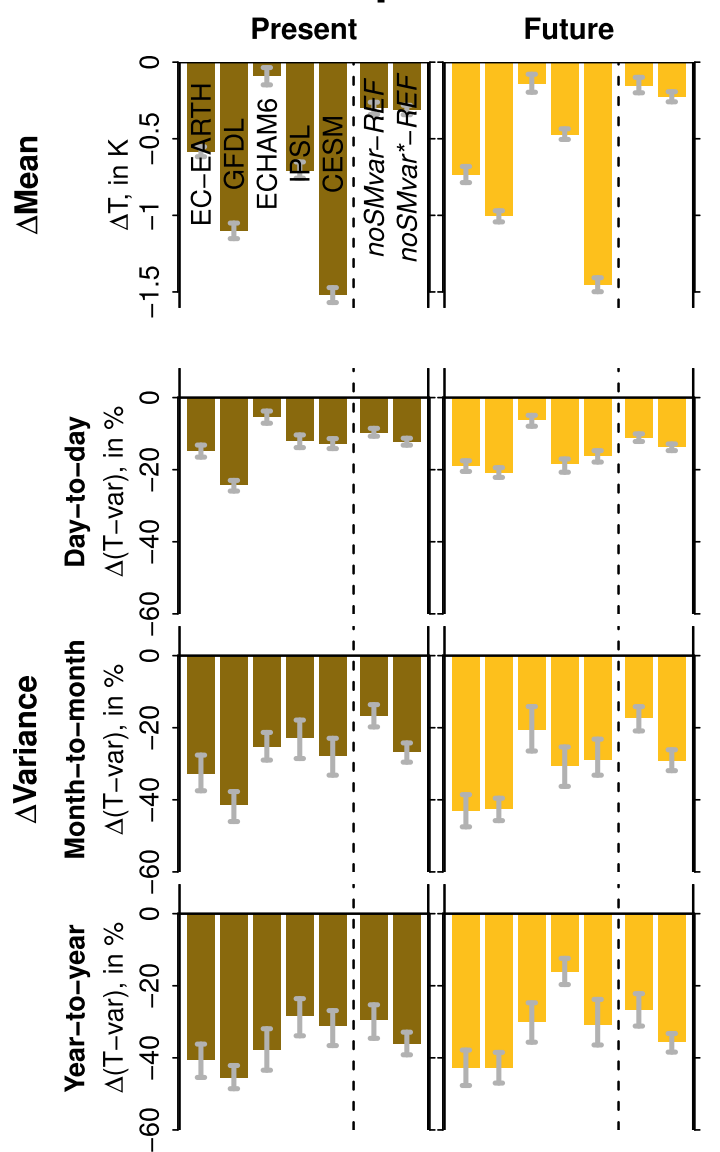

Precipitation
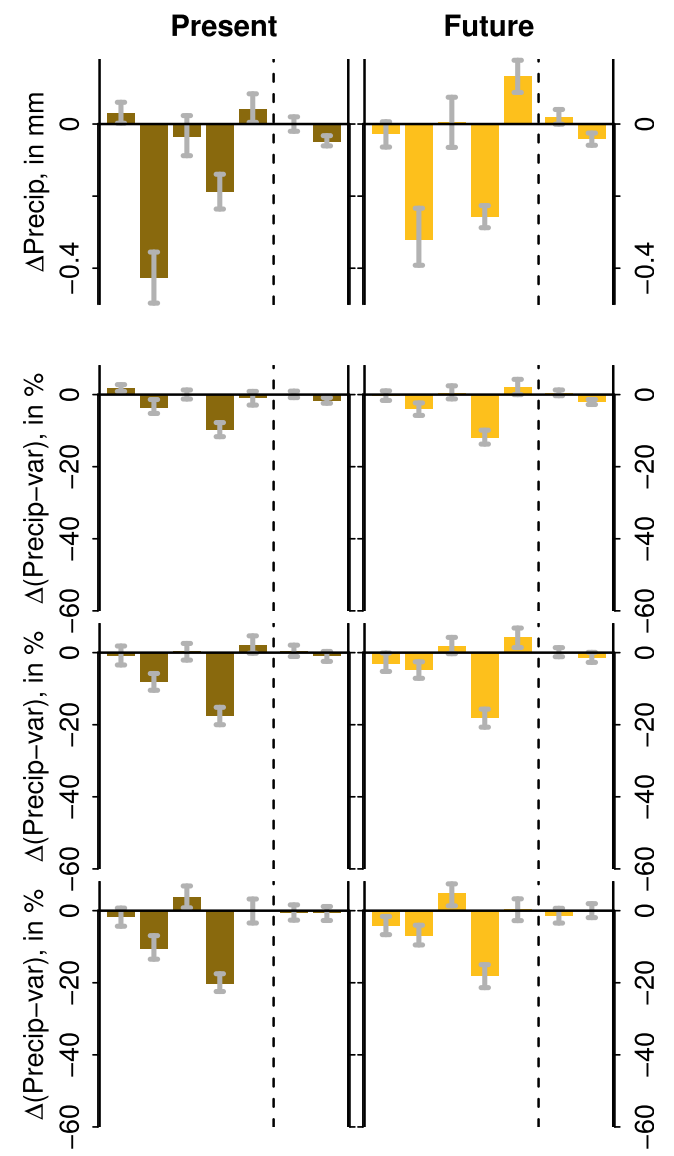

FIG. 13. Comparing the impact of removed SM variability in multimodel results of the GLACE-CMIP5 experiment with respective findings from this study; (top) changes in land climate mean and (bottom) variability changes (different time scales). Results are shown for present and future periods analyzed over land areas from $65^{\circ} \mathrm{N}-65^{\circ} \mathrm{S}$. Whiskers indicate the $5 \%-95 \%$ confidence range.

differences between GCMs. For future studies with strong computational constraints, it might therefore be an option to prescribe an interannually varying ocean rather than computing it interactively. On the other hand, an interactive ocean is required in other analyses, such as investigations related to impacts on the global carbon cycle.

Interestingly, the results of this analysis of the multimodel GLACE-CMIP5 database confirm other SMrelated findings of our study [which were in part also reported in Seneviratne et al. (2013) and Berg et al. (2014)]: (i) land climate response to prescribed SM is similar between the present and future periods, and (ii) the variability of temperature is more strongly reduced than that of precipitation, and the reduction is strongest at the interannual time scale.

\section{c. Novel findings in this study}

Many modeling studies have explored and compared the roles of SSTs and SM in coupled GCMs (Koster et al. 2000; Dirmeyer 2001; Reale and Dirmeyer 2002; Reale et al. 2002, Dirmeyer 2003). Using simulations from state-of-the-art ESMs, we confirm their results and find overall a similar influence of SM on land climate to that of SSTs. Our results show generally stronger changes in land climate variability in the tropics compared with the subtropics and midlatitudes (except for the impact of SM on precipitation variability, which is rather similar across latitudes), whereas changes in land climate means are strongest in the midlatitudes. Such contrasting results for changes in mean and variance of land climate are in line with the results of Reale and Dirmeyer (2002). Furthermore, our results confirm earlier findings in Koster et al. (2000), who reported the strongest impact of SSTs on precipitation variability in the tropics. Koster et al. (2000) furthermore show a strong role of SM for precipitation variability in the midlatitudes, which, however, is not in agreement with our results. In 
contrast to most of the previous studies, we find smaller changes in precipitation variability in response to removed SM or SST variability; in many regions, they cannot be separated from random noise, as shown by the significance assessment.

The hypothesis from Koster et al. (2000), which states that land-atmosphere interactions linearly amplify precipitation variance induced by the ocean and the atmosphere, is generally found not to be applicable. This was also found by Reale et al. (2002) and is here confirmed by our results showing that the land- and ocean-induced fractions of precipitation variance are mostly independent, except for regions where SSTs are influencing (land) precipitation and, hence, SM. This independence implies that land- and ocean-induced components of precipitation variability are mostly additive, a behavior found in our analysis when comparing the noALLvar experiments with the noSMvar and noOCNvar experiments.

In addition, the present study includes several new features:

- Comprehensive analysis of means and variabilities of precipitation and temperature

- Investigation of different deciles of precipitation and temperature (changes)

- Comparison of results for present versus future climate

- Assessment of model dependency of impact of removed SM variability on climate

In summary, our results highlight the main novel aspects: 1) similar magnitude and some degree of independence of SST and SM effects for temperature; 2) possibly smaller (and mostly insignificant) effect on precipitation compared with previous studies; 3) geographically no strong distinction in the response of land climate to SM versus SSTs in the warm season (i.e., no preferred latitudinal band for a dominant SM or SST effect).

\section{Conclusions}

In this study, we compare the impacts of SM versus SST variability on land climate. For this purpose, we used the global climate model CESM to perform ensemble simulations with prescribed SM and SST in which we removed their variability, respectively. These simulations are then compared with fully interactive reference simulations to assess the SM- or SST-related changes on land climate means and variability. Compared to previous studies on SM-related feedbacks, our main purpose is the assessment of the respective strength of the SM-climate coupling in comparison with that of the SST-climate coupling. The main result of this study is that SM variability has globally a similar impact on land climate as SST variability during the warm season and that both act as largely independent drivers of land climate means and variabilities. For mean climate, these results are consistent with findings from previous studies (Koster et al. 2000; Dirmeyer 2001; Reale and Dirmeyer 2002; Reale et al. 2002; Dirmeyer 2003). But to our knowledge, this is the first study to also provide a corresponding analysis for the whole temperature and precipitation distributions. The results show that high extremes of temperature and precipitation tend to be more strongly affected by both SM and SST variability. In addition, we analyze the feedbacks in both present and future climate and find overall similar features in the CESM. Finally, we note that we used a fully coupled model setting (i.e., coupled ocean), which was not the case of previous studies on this topic. We summarize hereafter a few additional results.

In terms of land temperature variability, we find stronger changes at larger temporal scales and toward lower latitudes from both SM and SST variability removal, whereas the corresponding means change more toward higher latitudes in the case of SM variability removal. The cooling in response to removed SM variability can be explained with the shape of the relationship between SM and evaporative fraction; they are independent under wet conditions and linearly related under drier conditions such that when prescribing SM to the median the wettening of dry soils leads to a cooling, whereas the drying of wet soils leads to no or minor warming (unlike in the case of drying of dry soils). Changes in precipitation variabilities and means, and of drought length (maximum amount cumulative dry days per year) in response to removed SM or SSTs are insignificant in many regions, especially in higher latitudes. The contrasting magnitude of the temperature and precipitation/drought changes may be explained by the fact that precipitation formation is influenced by various processes across the entire atmospheric column, whereas we consider temperature changes $2 \mathrm{~m}$ above the surface, which is close to where we apply the SM and SST changes. Overall land climate variabilities drop by $10 \%-50 \%$ for temperature and by $0 \%-10 \%$ for precipitation in response to either removed SM or SST variability (see Fig. 10). Changes are stronger for hot temperatures, for extreme precipitation, and for long droughts, particularly in the case of removed SM variability. This asymmetry is present across latitudes. These impacts on land climate are similar between the present climate and a warmer climate at the end of the century in the case of the RCP8.5 emissions scenario, except for smaller 
temperature changes following the removal of SM variability at the end of the century. Note here that especially the results for land climate means are sensitive to the prescribed SST fields (median vs neutral SSTs); similarly, they could be sensitive to the prescribed SM fields (not tested).

Furthermore we identify controls of the SM- and SST-induced climate changes. Impacts of removed SM variability at the local scale depend partly on (i) the reference SM variability and (ii) the strength of the land-atmosphere coupling (i.e., by the amount of variability removed and by the strength of the SMtemperature relationship). The SST influence is weakly related with the strength of the coupling between local precipitation or temperature and ENSO. However, these controls cannot explain all impacts on climate, partly because of various indirect effects: (i) the nonlocal effects of SM on land climate and (ii) other indirect effects. These include SST-induced variations in SM, which indirectly trigger impacts on land climate and, to a smaller extent, also SM-induced variations in SSTs (which may also feed back on land climate). Other controls that are not considered in this study probably also play a role.

The main indirect effects result from interdependencies between SM and SSTs. As we aim in this study to compare their specific impacts, we investigate if they are sufficiently independent. For this purpose, we analyze the sum of the SM- and SST-induced climate changes, respectively, and find that they scale well with the impact of removing both variabilities together; this is the case in terms of land climate means but to a lesser extent for climate variability. To test the robustness of our conclusions given these interdependencies, we repeat the model experiments with removed SM and SST variability, respectively, but with prescribed SST and SM from the reference experiment, respectively, thereby preventing any interdependencies. The results are generally similar, thus indicating a large level of independence between SM and SST dynamics (in CESM). More importantly, the results show that SM does not (only) act as an amplifier of SST-induced anomalies but mostly independently of the latter.

We also analyze if our results are generally valid irrespective of the choice of the model. For this purpose, we compare the land climate response to removed SM variability between our experiments and similar experiments performed within the GLACE-CMIP5 project, which involved five GCMs that performed experiments similar to noSMvar*. We find similar results across the five models and our experiments, which underlines that our findings are not strongly dependent on the employed model.
In conclusion, our results confirm the important role of land hydrology for temperature and precipitation during the warm season and underline that SM (variability) can and does impact land climate independent of (the variability of) SSTs. Since SM is comparatively soundly predictable thanks to its memory characteristics (Koster et al. 2011; Seneviratne and Koster 2012; Orth and Seneviratne 2012), our findings underline the need for a careful consideration of initial SM as an independent predictor-similar to the well-established consideration of initial SST conditions-in weather and climate forecasting over land. This is even more important in the case of extreme events, such as heat waves, for which the accurate representation of SM can add yet more predictive skill.

Acknowledgments. We thank Urs Beyerle, Jan Sedlacek, Erich Fischer, and Mathias Hauser for support with the CESM simulations and helpful discussions on the experiment design. We also thank Paul Dirmeyer and another anonymous reviewer for helpful comments on the manuscript. We acknowledge the GLACE-CMIP5 modeling groups for the simulations. We acknowledge funding from the European Union's Seventh Framework Programme (FP7/2007-13) under Grants 607085 (EUCLEIA) and 282769 (DROUGHT-R\&SPI), as well as the European Research Council under Grant 617518 (DROUGHT-HEAT).

\section{REFERENCES}

Berg, A., B. Lintner, K. Findell, S. Malyshev, P. Loikith, and P. Gentine, 2014: Impacts of soil moisture-atmosphere interactions on surface temperature distribution. J. Climate, 27, 7976-7993, doi:10.1175/JCLI-D-13-00591.1.

Bichet, A., M. Wild, D. Folini, and C. Schär, 2011: Global precipitation response to changing forcings since 1870. Atmos. Chem. Phys., 11, 9961-9970, doi:10.5194/acp-11-9961-2011.

Chung, C. T. Y., S. B. Power, J. M. Arblaster, H. A. Rashid, and G. L. Roff, 2014: Nonlinear precipitation response to El Niño and global warming in the Indo-Pacific. Climate Dyn., $\mathbf{4 2}$, 1837-1856, doi:10.1007/s00382-013-1892-8.

Dai, A., and T. M. L. Wigley, 2000: Global patterns of ENSOinduced precipitation. Geophys. Res. Lett., 27, 1283-1286, doi:10.1029/1999GL011140.

Dirmeyer, P. A., 2001: An evaluation of the strength of landatmosphere coupling. J. Hydrometeor., 2, 329-344, doi:10.1175/ 1525-7541(2001)002<0329:AEOTSO > 2.0.CO;2.

- 2003: The role of the land surface background state in climate predictability. J. Hydrometeor., 4, 599-610, doi:10.1175/ 1525-7541(2003)004<0599:TROTLS>2.0.CO;2.

- 2011: The terrestrial segment of soil moisture-climate coupling. Geophys. Res. Lett., 38, L16702, doi:10.1029/2011GL048268.

_ - Y. Jin, B. Singh, and X. Yan, 2013a: Trends in land-atmosphere interactions from CMIP5 simulations. J. Hydrometeor., 14, 829 849, doi:10.1175/JHM-D-12-0107.1.

_ S. Kumar, M. J. Fennessy, E. L. Altshuler, T. DelSole, Z. Guo, B. A. Cash, and D. Straus, 2013b: Model estimates of 
land-driven predictability in a changing climate from CCSM4. J. Climate, 26, 8495-8512, doi:10.1175/JCLI-D-13-00029.1.

Douville, H., 2003: Assessing the influence of soil moisture on seasonal climate variability with AGCMs. J. Hydrometeor., 4, 1044-1066, doi:10.1175/1525-7541(2003)004<1044:ATIOSM>2.0.CO;2.

_- J. Colin, E. Krug, J. Cattiaux, and S. Thao, 2016: Midlatitude daily summer temperatures reshaped by soil moisture under climate change. Geophys. Res. Lett., 43, 812-818, doi:10.1002/ 2015 GL066222.

Ek, M. B., and A. A. M. Holtslag, 2004: Influence of soil moisture on boundary layer cloud development. J. Hydrometeor., 5, 86-99, doi:10.1175/1525-7541(2004)005<0086:IOSMOB>2.0.CO;2.

Gates, W. L., 1992: AMIP: The Atmospheric Model Intercomparison Project. Bull. Amer. Meteor. Soc., 73, 1962-1970, doi:10.1175/1520-0477(1992)073<1962:ATAMIP>2.0.CO;2.

Gent, P. R., and Coauthors, 2011: The Community Climate System Model version 4. J. Climate, 24, 4973-4991, doi:10.1175/ 2011JCLI4083.1.

Giannini, A., R. Saravanan, and P. Chang, 2003: Oceanic forcing of Sahel rainfall on interannual to interdecadal time scales. Science, 302, 1027-1030, doi:10.1126/science.1089357.

Guillod, B. P., B. Orlowsky, D. G. Miralles, A. J. Teuling, and S. I. Seneviratne, 2015: Reconciling spatial and temporal soil moisture effects on afternoon rainfall. Nat. Commun., 6, 6443 doi:10.1038/ncomms 7443 .

Guo, Z., and Coauthors, 2006: GLACE: The Global LandAtmosphere Coupling Experiment. Part II: Analysis. J. Hydrometeor., 7, 611-625, doi:10.1175/JHM511.1.

Hack, J. J., 1994: Parameterization of moist convection in the National Center for Atmospheric Research Community Climate Model (CCM2). J. Geophys. Res., 99, 5551-5568, doi:10.1029/93JD03478.

Hirschi, M., and Coauthors, 2011: Observational evidence for soilmoisture impact on hot extremes in southeastern Europe. Nat. Geosci., 4, 17-21, doi:10.1038/ngeo1032.

Hoerling, M., J. Hurrell, J. Eischeid, and A. Phillips, 2006: Detection and attribution of twentieth-century northern and southern African rainfall change. J. Climate, 19, 3989-4008, doi:10.1175/JCLI3842.1.

Hong, S.-Y., and E. Kalnay, 2000: Role of sea surface temperature and soil-moisture feedback in the 1998 Oklahoma-Texas drought. Nature, 408, 842-844, doi:10.1038/35048548.

Hoskins, B. J., and D. J. Karoly, 1981: The steady linear response of a spherical atmosphere to thermal and orographic forcing. J. Atmos. Sci., 38, 1179-1196, doi:10.1175/ 1520-0469(1981)038<1179:TSLROA > 2.0.CO;2.

Jaeger, E. B., and S. I. Seneviratne, 2011: Impact of soil moistureatmosphere coupling on European climate extremes and trends in a regional climate model. Climate Dyn., 36, 19191936, doi:10.1007/s00382-010-0780-8.

Jin, E. K., and J. L. Kinter, 2009: Characteristics of tropical Pacific SST predictability in coupled GCM forecasts using the NCEP CFS. Climate Dyn., 32, 675-691, doi:10.1007/s00382-008-0418-2.

Koster, R. D., and M. J. Suarez, 1995: Relative contributions of land and ocean processes to precipitation variability. J. Geophys. Res., 100, 13 775-13 790, doi:10.1029/95JD00176.

— , and —, 2001: Soil moisture memory in climate models. J. Hydrometeor., 2, 558-570, doi:10.1175/1525-7541(2001)002<0558: $\mathrm{SMMICM}>2.0 . \mathrm{CO} ; 2$.

_ - — , and M. Heiser, 2000: Variance and predictability of precipitation at seasonal-to-interannual timescales. J. Hydrometeor., 1, 26-46, doi:10.1175/1525-7541(2000)001<0026: VAPOPA $>2.0 . \mathrm{CO} ; 2$.
_ and Coauthors, 2004: Regions of strong coupling between soil moisture and precipitation. Science, 305, 1138-1140, doi:10.1126/ science. 1100217.

— and Coauthors, 2011: The second phase of the Global LandAtmosphere Coupling Experiment: Soil moisture contributions to subseasonal forecast skill. J. Hydrometeor., 12, 805-822, doi:10.1175/2011JHM1365.1.

- Y. Chang, and S. D. Schubert, 2014: A mechanism for landatmosphere feedback involving planetary wave structures. J. Climate, 27, 9290-9301, doi:10.1175/JCLI-D-14-00315.1.

Mueller, B., and S. I. Seneviratne, 2012: Hot days induced by precipitation deficits at the global scale. Proc. Natl. Acad. Sci. USA, 109, 12 398-12 403, doi:10.1073/pnas.1204330109.

Neale, R. B., J. H. Richter, and M. Jochum, 2008: The impact of convection on ENSO: From a delayed oscillator to a series of events. J. Climate, 21, 5904-5924, doi:10.1175/2008JCLI2244.1.

— , and Coauthors, 2010: Description of the NCAR Community Atmosphere Model (CAM 4.0). NCAR Tech. Note NCAR/TN-485+STR, 224 pp. [Available online at http:// www.cesm.ucar.edu/models/ccsm4.0/cam/docs/description/ cam4_desc.pdf.]

Oleson, K. W., and Coauthors, 2010: Technical description of version 4.0 of the Community Land Model (CLM). NCAR Tech. Note NCAR/TN-478+STR, 266 pp. [Available online at http://www.cesm.ucar.edu/models/ccsm4.0/clm/CLM4_ Tech_Note.pdf.]

Orth, R., and S. I. Seneviratne, 2012: Analysis of soil moisture memory from observations in Europe. J. Geophys. Res., 117, D15115, doi:10.1029/2011JD017366.

- and - 2013: Predictability of soil moisture and streamflow on sub-seasonal timescales: A case study. J. Geophys. Res., 118, 10 963-10 979, doi:10.1002/jgrd.50846.

_, and _ 2014: Using soil moisture forecasts for sub-seasonal summer temperature predictions in Europe. Climate Dyn., 43, 3403-3418, doi:10.1007/s00382-014-2112-x.

Rasmusson, E. M., and T. H. Carpenter, 1982: Variations in tropical sea surface temperature and surface wind fields associated with the Southern Oscillation/El Niño. Mon. Wea. Rev., 110, 354 383, doi:10.1175/1520-0493(1982)110<0354:VITSST >2.0.CO;2.

Reale, O., and P. Dirmeyer, 2002: Modeling the effect of land surface evaporation variability on precipitation variability. Part I: General response. J. Hydrometeor., 3, 433-450, doi:10.1175/1525-7541(2002)003<0433:MTEOLS > 2.0.CO;2.

, -, and A. Schlosser, 2002: Modeling the effect of land surface evaporation variability on precipitation variability. Part II: Time- and space-scale structure. J. Hydrometeor., 3, 451-466, doi:10.1175/1525-7541(2002)003<0451:MTEOLS > 2.0.CO;2.

Schubert, S. D., M. J. Suarez, P. J. Pegion, R. D. Koster, and J. T. Bacmeister, 2004: On the cause of the 1930s Dust Bowl. Science, 303, 1855-1859, doi:10.1126/science.1095048.

Seneviratne, S. I., and R. D. Koster, 2012: A revised framework for analyzing soil moisture memory in climate data: Derivation and interpretation. J. Hydrometeor., 13, 404-412, doi:10.1175/ JHM-D-11-044.1.

, D. Lüthi, M. Litschi, and C. Schär, 2006a: Land-atmosphere coupling and climate change in Europe. Nature, 443, 205-208, doi:10.1038/nature05095.

, and Coauthors, 2006b: Soil moisture memory in AGCM simulations: Analysis of Global Land-Atmosphere Coupling Experiment (GLACE) data. J. Hydrometeor., 7, 1090-1112, doi:10.1175/JHM533.1.

, T. Corti, E. L. Davin, M. Hirschi, E. B. Jaeger, I. Lehner, B. Orlowsky, and A. J. Teuling, 2010: Investigating 
soil moisture-climate interactions in a changing climate: A review. Earth Sci. Rev., 99, 125-161, doi:10.1016/ j.earscirev.2010.02.004.

_, and Coauthors, 2013: Impact of soil moisture-climate feedbacks on CMIP5 projections: First results from the GLACE-CMIP5 experiment. Geophys. Res. Lett., 40, 5212-5217, doi:10.1002/ grl.50956.

Shukla, J., 1998: Predictability in the midst of chaos: A scientific basis for climate forecasting. Science, 5389, 728-731, doi:10.1126/ science.282.5389.728.

Smith, R., and Coauthors, 2010: The Parallel Ocean Program (POP) reference manual. UCAR Tech. Rep. LAUR-10-01853, $141 \mathrm{pp}$. [Available online at http://www.cesm.ucar.edu/models/cesm1.1/ pop2/doc/sci/POPRefManual.pdf.]

Taylor, C. M., A. Gounou, F. Guichard, P. P. Harris, R. J. Ellis, F. Couvreux, and M. D. Kauwe, 2011: Frequency of Sahelian storm initiation enhanced over mesoscale soil-moisture patterns. Nat. Geosci., 4, 430-433, doi:10.1038/ngeo1173.
Teuling, A. J., and Coauthors, 2009: A regional perspective on trends in continental evaporation. Geophys. Res. Lett., 36, L02404, doi:10.1029/2008GL036584.

Whan, K., J. Zscheischler, R. Orth, M. Shongwe, M. Rahimi, E. Asare, and S. I. Seneviratne, 2015: Impact of soil moisture on extreme maximum temperatures in Europe. Wea. Climate Extremes, 9, 57-67, doi:10.1016/j.wace.2015.05.001.

Wu, R., and J. L. Kinter, 2009: Analysis of the relationship of U.S. droughts with SST and soil moisture: Distinguishing the time scale of droughts. J. Climate, 22, 4520-4538, doi:10.1175/ 2009JCLI2841.1.

Zhou, T., R. Yu, H. Li, and B. Wang, 2008: Ocean forcing to changes in global monsoon precipitation over the recent half-century. J. Climate, 21, 3833-3852, doi:10.1175/2008JCLI2067.1.

Zscheischler, J., R. Orth, and S. I. Seneviratne, 2015: A sub-monthly database for detecting changes in vegetation-atmosphere coupling. Geophys. Res. Lett., 42, 9816-9824, doi:10.1002/ 2015 GL066563. 